\title{
Advances in the controlled polymerization of conjugated
}

\section{polymers}

Lize Verheyen, Pieter Leysen, Marie-Paule Van Den Eede, Ward Ceunen, Tine Hardeman, Guy Koeckelberghs*

Laboratory for Polymer Synthesis, KU Leuven, Celestijnenlaan 200F, B-3001 Heverlee (Belgium)

guy.koeckelberghs@chem.kuleuven.be

\begin{abstract}
This article features recent advances in the synthesis of conjugated polymers via a controlled polymerization. These polymerizations typically rely on transition metal catalyzed cross coupling reactions. The mechanisms of the polymerization protocols are discussed in detail. An overview of all possible protocols and all homopolymers that have been investigated is given. Next, the synthesis of copolymers - random, gradient and block copolymers - is reviewed. Another advantage of a controlled polymerization is the possibility to introduce specific functional groups, either at the beginning of each polymer chain by the use of an external initiator, or at the end of the polymer chain using an endcapper. Finally, topologies different from simple linear polymer chains are discussed. This feature article is complementary to other recent review articles on this topic. ${ }^{1,2}$
\end{abstract}

\section{Introduction}

Almost all conjugated polymers (CPs) are prepared using transition metal catalyzed cross coupling reactions. In short, these reactions consists of an oxidative addition (OA), followed by a transmetalation (TM) and, finally, a reductive elimination (RE), after which the cycle 
restarts (Scheme 1, black arrows). Such polymerizations are clearly polycondensations and one can assume that those polymerizations proceed in a step-growth fashion. However, in 2004, Yokozawa ${ }^{3}$ and McCullough $^{4}$ independently discovered that a particular polymerization, i.e. poly(3-alkylthiophene) (P3AT) obtained with a $\mathrm{Ni}(\mathrm{dppp}) \mathrm{Cl}_{2}$ catalyst $(\mathrm{dppp}=1,3$-bis(diphenylphosphino)propane), proceeds in a controlled chain-growth fashion. This discovery marked the beginning of the exploration of the controlled nature of the polymerization of CPs. In general, there are two ways to realize a controlled polymerization of CPs. The first and by far most used way relies on the complexation of the catalyst to the $\pi$ system of the growing polymer chain. In this way the catalyst remains complexed to the growing polymer chain after reductive elimination and is transferred to a terminal $\mathrm{C}$-Br bond where it oxidatively inserts. This type of polymerization is called a catalyst transfer polymerization (CTP). Termination can occur if the catalyst diffuses away prior to oxidative addition or by disproportionation. If termination and transfer reactions are retarded, one catalyst/initiator moiety polymerizes one polymer chain and a controlled polymerization is realized. The association of the catalyst with the polymer chain is crucial. The first evidence of the existence of this complex was provided by McNeil, who found that the presence of activated aryl halides does not affect the polymerization, proving that the catalyst does not dissociate from the growing polymer chain. ${ }^{5}$ Later, Kiriy found ${ }^{31} \mathrm{P}$ NMR signals that could be attributed to the complex in the polymerization of a $\mathrm{CP}$ that proceeds via a Ni-catalyst, but not in a classical oxidative addition, transmetalation and reductive elimination fashion. ${ }^{6}$ Finally, our group has demonstrated the existence of this complex in an attempt to polymerize thienothiophenes; in fact, the stability of the complex hampered the polymerization of this monomer. ${ }^{7}$ Several coupling reactions have been utilized in CTP, including the initially investigated Kumada catalyst transfer polymerization (KCTP) that uses Kumada couplings, Suzuki-Miyaura catalyst transfer polymerization (SCTP) using the Suzuki-Miyaura reaction ${ }^{8}$ 
and, more recently, CTPs that exploit Sonogashira ${ }^{9}$, Stille $^{9,10}$, Negishi $^{4}$ and Murahashi ${ }^{11}$ couplings.

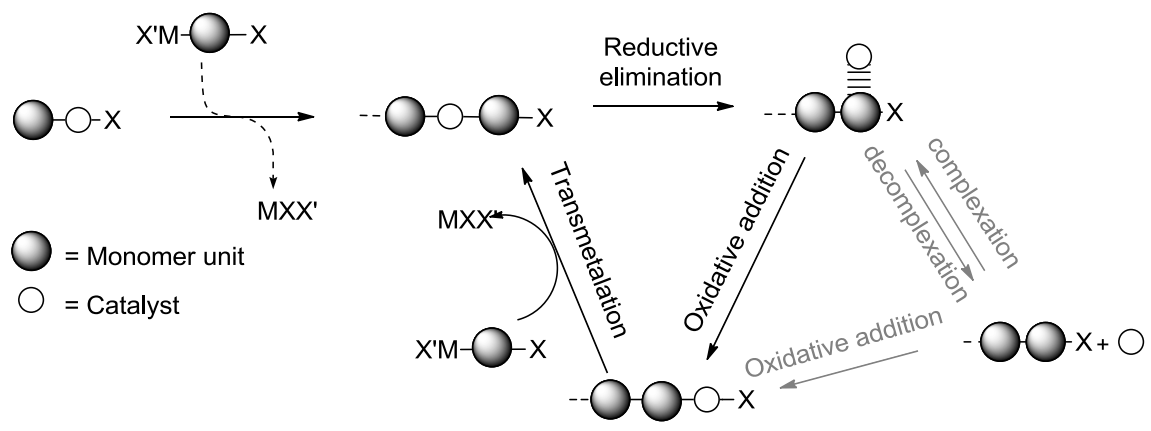

Scheme 1: Two ways to realize a controlled polymerization of CPs.

An interesting tool to screen different catalysts for a CTP is the reaction with a corresponding monomer bearing two halides instead of one. If the catalyst remains complexed after one reaction, it will perform a second, intramolecular oxidative insertion rather than diffusing away. The result is that either no capping or dicapping occurs, but no monocapping. Monocapping requires that the catalyst dissociates after one reaction, which does not occur in efficient CTP. As a result, this test can be used to screen reaction conditions for efficient CTP. ${ }^{5,12,13}$ Importantly, the screening must be performed at low conversions. If the halide becomes more reactive after one reaction and the catalyst does dissociate, monocapped product will indeed be present at low conversions. However, since it is more reactive than the starting product, it will vanish at higher conversions because reaction rather occurs on monocapped product. $^{14}$

A second way to realize a controlled polymerization of CPs is inspired by Yokozawa's controlled polymerization of aromatic amides. ${ }^{15}$ It uses AB-type monomers and the key to success is the deactivation of one functional group by the other in the monomer. However, after reaction this deactivation is lost. As a consequence, growth is only possible on the growing polymer chains (Scheme 1, grey arrows). The catalyst can decomplex, but, since 
reaction with monomer is impossible, it must perform an oxidative addition on a (dormant) polymer chain and restart the polymerization. The choice of the catalyst is crucial: it must be stable when dissociated and oxidatively insert very easily into a dormant polymer chain. The catalyst that has been used is $\operatorname{Pd}\left(\right.$ Ruphos). ${ }^{16}$ The advantage of this procedure is that the controlled nature of the polymerization does not depend on the complexation of the catalyst with the growing polymer chain, which is system-dependent. Moreover, the independence of this complexation has also additional advantages, like the more easily formation of block and gradient copolymers (see section 3 . Monomer deactivation). The dissociation of the catalyst is demonstrated by the addition of an aryl halide, which acts as a transfer reagent, limiting the degree of polymerization. ${ }^{16}$

\section{Catalyst transfer polymerizations}

\subsection{Initiation}

Since the KCTP of P3AT is by far the most used and investigated CTP, the basic principles of initiation will be explained based on this system. ${ }^{3,4,15,17-25}$ As can be seen in Scheme 2, the initiation starts with two consecutive transmetalations, in which two halogens on the catalyst are exchanged for two monomeric units. After those transmetalations, reductive elimination takes place and a tail-to-tail dimer is formed. Due to the complexation of the catalyst to this dimer after reductive elimination, the following oxidative addition will occur intramolecularly in one of the two terminal C-Br bonds. The same three initiation steps can also be found for $\mathrm{PdL}_{2} \mathrm{X}_{2}$ initiators and in other CTPs based on Suzuki-Miyaura ${ }^{26}, \mathrm{Negishi}^{4}$, Murahashi $^{11}$ or Stille ${ }^{10}$ couplings. 

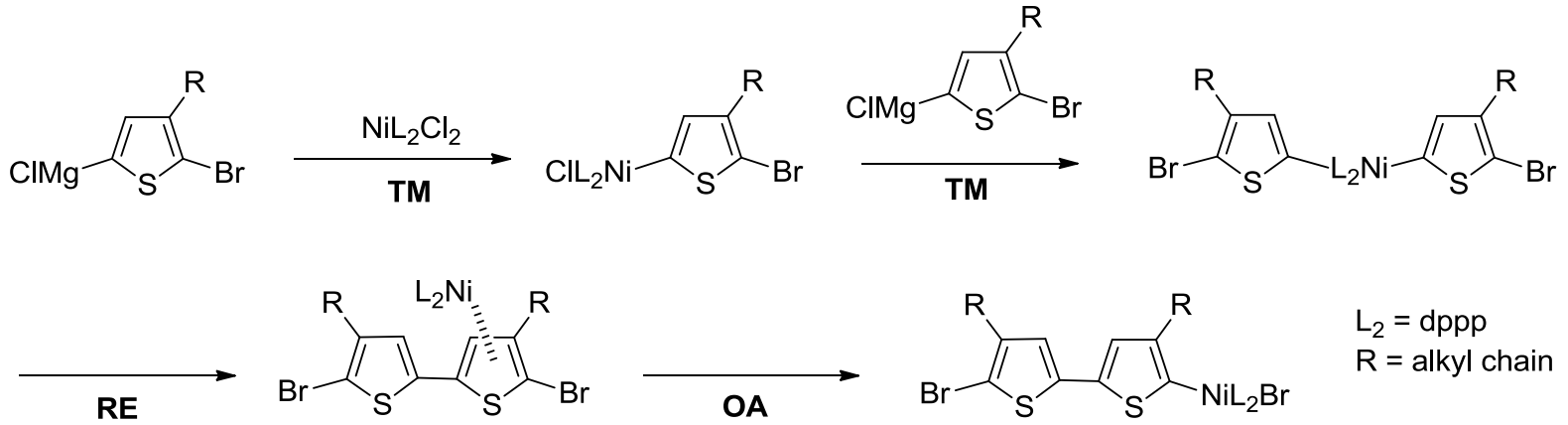

Scheme 2: Initiation in KCTP of P3AT with $\mathrm{Ni}(\mathrm{dppp}) \mathrm{Cl}_{2}$.

\subsubsection{External initiators}

Instead of using $\mathrm{Ni}(\mathrm{L})_{2} \mathrm{X}_{2}$ or $\mathrm{Pd}(\mathrm{L})_{2} \mathrm{X}_{2}$ as catalyst, as shown above, it is also possible to work with external initiators. In this way, functional groups can easily be incorporated at the beginning of the polymer chain, which makes this the most popular technique to incorporate functional groups into conjugated polymers. Even if the polymerization is not controlled, all polymer chains will still be equipped with the functional group in the beginning, as long as no transfer reactions occur. The initiating steps using external initiators are similar to those with $\mathrm{Ni}(\mathrm{L})_{2} \mathrm{X}_{2}$ or $\mathrm{Pd}(\mathrm{L})_{2} \mathrm{X}_{2}$, but due to the presence of a reactive ligand, only transmetalation with one monomer is necessary (Scheme 3). After this step, the initiation proceeds in the same way as with $\mathrm{Ni}(\mathrm{L})_{2} \mathrm{X}_{2}$ or $\mathrm{Pd}(\mathrm{L})_{2} \mathrm{X}_{2}$ : first a reductive elimination, after which the catalyst stays complexed to the dimer, followed by an intramolecular oxidative addition. However, since there is usually no halogen present on the reactive ligand, the catalyst can only insert into the $\mathrm{C}-\mathrm{Br}$ bond of the incorporated monomer and bidirectional growth is prevented. This is also the reason why external initiators are often used in the synthesis of block copolymers (see 2.2.8. All-conjugated block copolymers). 


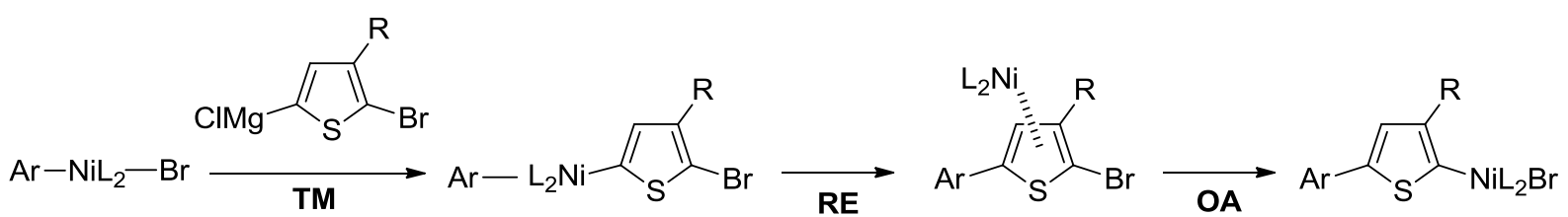

Scheme 3: Initiation in KCTP of P3AT with an external initiator.

The first generation of external Ni-initiators, used in the polymerization of P3ATs or other CPs, were prepared in situ right before their use in the polymerization. Different synthetic routes were investigated and the first one was the oxidative addition of $\mathrm{Ni}\left(\mathrm{PPh}_{3}\right)_{4}$ in the desired aryl bromide, resulting in $(\mathrm{Ar}) \mathrm{Ni}\left(\mathrm{PPh}_{3}\right)_{2} \mathrm{Br}^{27-29}$ However, the success of this oxidative addition depends on the nature and the position of the functional groups on the reactive ligand ${ }^{27,30,31}$ and due to the air sensitive and carcinogenic character of $\mathrm{Ni}\left(\mathrm{PPh}_{3}\right)_{4}$ also other methods were tried. It is for example possible to combine an aryl bromide with BuLi followed by the addition of $\mathrm{Ni}\left(\mathrm{PPh}_{3}\right)_{2} \mathrm{Cl}_{2}{ }^{27}$ In this way $\mathrm{Ni}\left(\mathrm{PPh}_{3}\right)_{2} \mathrm{Cl}_{2}$, which is air stable, can be used as nickel source.

The previous methods utilize monodentate $\mathrm{PPh}_{3}$ as ligand, but after the group of Kiriy discovered that bidentate ligands, like dppp and dppe (1,3-bis(diphenylphosphino)ethane), give rise to a better control over the polymerization, other methods were tried to incorporate those bidentate ligands. ${ }^{28,29}$ The first method was based on the ligand exchange between $\mathrm{PPh}_{3}$ and dppp or dppe on $(\mathrm{Ar}) \mathrm{Ni}\left(\mathrm{PPh}_{3}\right)_{2} \mathrm{Br}$, which could be synthesized with one of the methods mentioned above, and resulted in $(\mathrm{Ar}) \mathrm{Ni}\left(\mathrm{L}^{\prime}\right) \mathrm{Br}\left(\mathrm{L}^{\prime}=\right.$ bidentate ligand $) .{ }^{30,32,33}$ Another method is based on the combination of $\mathrm{Ni}$ (bipy)Et 2 (bipy $=2,2^{\prime}$-bipyridine) with the desired aryl halide, forming (Ar)Ni(bipy)Br. In a second step, a ligand exchange with a bidentate phosphorous ligand is performed, which results in $(\mathrm{Ar}) \mathrm{Ni}\left(\mathrm{L}^{\prime}\right) \mathrm{Br}^{20,34,35}$ The main drawbacks of this synthetic route are the high sensitivity of $\mathrm{Ni}$ (bipy)Et $\mathrm{Et}_{2}$ towards oxygen and moisture and the absence of commercial sources. It is also possible to combine $\mathrm{Ni}(\mathrm{COD})_{2}$ with a bidentate 
ligand, followed by reaction with an aryl halide ${ }^{36}$ or to perform a one-step reaction of $\mathrm{Ni}(\mathrm{dppp}) \mathrm{Cl}_{2}$ with an aryl magnesium chloride ${ }^{37,38}$. For the last method, only ortho-substituted aromatic compounds can be used, otherwise the homo-coupling of the aromatic units is possible. In addition to aromatic reactive ligands, an allyl functional group has also been used in external initiators. This $\pi$-allyl nickel complex was synthesized by combining bis(1,5cyclooctadiene)nickel and allyltrifluoroacetate, followed by the addition of dppp (or $\mathrm{PPh}_{3}$ ), resulting in (allyl) $\mathrm{Ni}(\mathrm{L}) \mathrm{OCOCF}_{3} .{ }^{39}$

The major drawback of all previously mentioned methods is the absence of a purification step, leaving impurities in the reaction mixture. Some of those impurities, such as $\mathrm{Ni}^{2+}$-salts, can also initiate the polymerization, creating polymer chains without the desired functional group at the beginning of the chain. To overcome this problem, new synthetic routes were developed and Scheme 4 summarizes all purified external nickel initiators that were synthesized up to now. Most of those initiators are synthesized with $\mathrm{PPh}_{3}$ as ligand and are purified in this form. Then, right before the polymerization, a ligand exchange with the desired ligand is performed. After this step, no additional purification is needed, because normally the exchange reaction is quantitative and free $\mathrm{PPh}_{3}$ has no influence on the polymerization. The stability of the purified initiator must however be checked, because some degradation products can also initiate the polymerization, yielding unfunctionalized chains. In our group, initiators $\mathbf{1}$ to $\mathbf{1 4}$ were synthesized, purified and used for the synthesis of P3ATs after they were subjected to a ligand exchange reaction with two equivalents of dppp (unless stated otherwise). Initiator $\mathbf{1}$, however, was unstable in solution, which led to the formation of $\mathrm{Ni}\left(\mathrm{PPh}_{3}\right)_{2} \mathrm{Br}_{2}$. This complex can initiate the polymerization on its own, creating unfunctionalized chains. ${ }^{31}$ Due to the ortho-substituent present in initiator $\mathbf{2}$, this complex is much more stabilized and no disproportionation occurs. It is suggested that the ortho-substituent increases the stability of the Ni-complex by lowering the energy of the HOMO due to bonding of the $\mathrm{d}_{\mathrm{xy}}$-orbitals of $\mathrm{Ni}$ 
with the $\pi$-orbitals of the aromatic ring. ${ }^{40}$ Later on it was shown that the ortho-stabilization is only necessary when monodentate ligands are used. ${ }^{21,41}$ Besides for $\mathrm{P}^{2} \mathrm{AT}^{31}$, initiator 2 was also used in the synthesis of poly(para-phenylene) (PPP) ${ }^{31}$ (1 equivalent of dppe was used for the ligand exchange) and poly(thienopyrazine) (PTP $)^{42}$. Initiators $\mathbf{3}$ to $\mathbf{1 3}$ were successfully used for the controlled synthesis of P3AT. ${ }^{31,43-49}$ In addition to the synthesis of P3AT, initiator 3 was also used for the synthesis of poly(3-alkoxythiophene) (P3AOT), although this was not controlled due to the intrinsic character of the alkoxythiophene monomer (see 2.2.1. Kumada catalyst transfer polymerization $(\mathrm{KCTP}))^{46,50}$ and initiator $\mathbf{1 2}$ was used in the synthesis of poly(3-alkylselenophene) $(\mathrm{P} 3 \mathrm{ASe})^{48}$. Polymerizations that were tried with initiator 14 were unsuccessful, probably due to the strong complexation of the Ni-catalyst to the reactive ligand, prohibiting normal propagation. ${ }^{45}$

Apart from the previous method, four other methods were found that can render purified external nickel initiators. In the group of Kiriy initiators $\mathbf{1 5}$ and $\mathbf{1 6}$ were synthesized by combining the corresponding aryl halide and $\mathrm{Ni}($ bipy)Et 2 , followed by a ligand exchange reaction with dppp and dppe, respectively. ${ }^{35,51}$ Also in the group of Bazan, initiator $\mathbf{1 5}$ was synthesized, but following a different synthetic route and with dppe as a ligand. First, $\mathrm{Ni}$ (dppe) $\mathrm{Cl}_{2}$ is combined with $\mathrm{PPh}_{3}$ in the presence of ethyl magnesium bromide, resulting in the formation of $\mathrm{Ni}(\mathrm{dppe})\left(\mathrm{PPh}_{3}\right)_{2}$, which was then reacted with bromobenzene. ${ }^{52}$ Initiator $\mathbf{1 7}$ was synthesized in the same way. ${ }^{53}$ Pammer et al. were able to polymerize thiazole monomers with initiators 18 and 19 , which were made by the sequential addition of $\mathrm{PPh}_{3}$, the desired aryl bromide and dppp to $\mathrm{Ni}(\mathrm{COD})_{2} .{ }^{54}$ The last method is based on the one-step reaction of $\mathrm{Ni}(\mathrm{dppp})_{2}$ with an aryl bromide, rendering (Ar)Ni(dppp)Br. Initiators $\mathbf{1 5}$ and $\mathbf{2 0}$ were synthesized in this way. ${ }^{41}$

Besides introducing functional groups, external initiators can also be used to influence the polymerization. The group of McNeil studied the influence of the reactive ligand on the 
polymerization of 4-bromo-2,5-bis(hexyloxy)phenylmagnesium chloride and found that changing this ligand could accelerate the initiation compared to the propagation, which led to a lower dispersity of the obtained polymers. ${ }^{55}$ It was found that reactive ligands with resonance-based electron-withdrawing substituents increase the initiation rate the most, since they stabilize the increasing electron density on the catalyst during the reductive elimination. Compared with the initiation by $\mathrm{Ni}(\mathrm{L})_{2} \mathrm{X}_{2}$, the use of external initiators increases the regioregularity and decreases the dispersity of the polymers, because the tail-to-tail dyad formed during the initiation is replaced by a head-to-tail dyad and the initiation is homogeneous compared to heterogeneous initiation with $\mathrm{Ni}(\mathrm{L})_{2} \mathrm{X}_{2}$. 

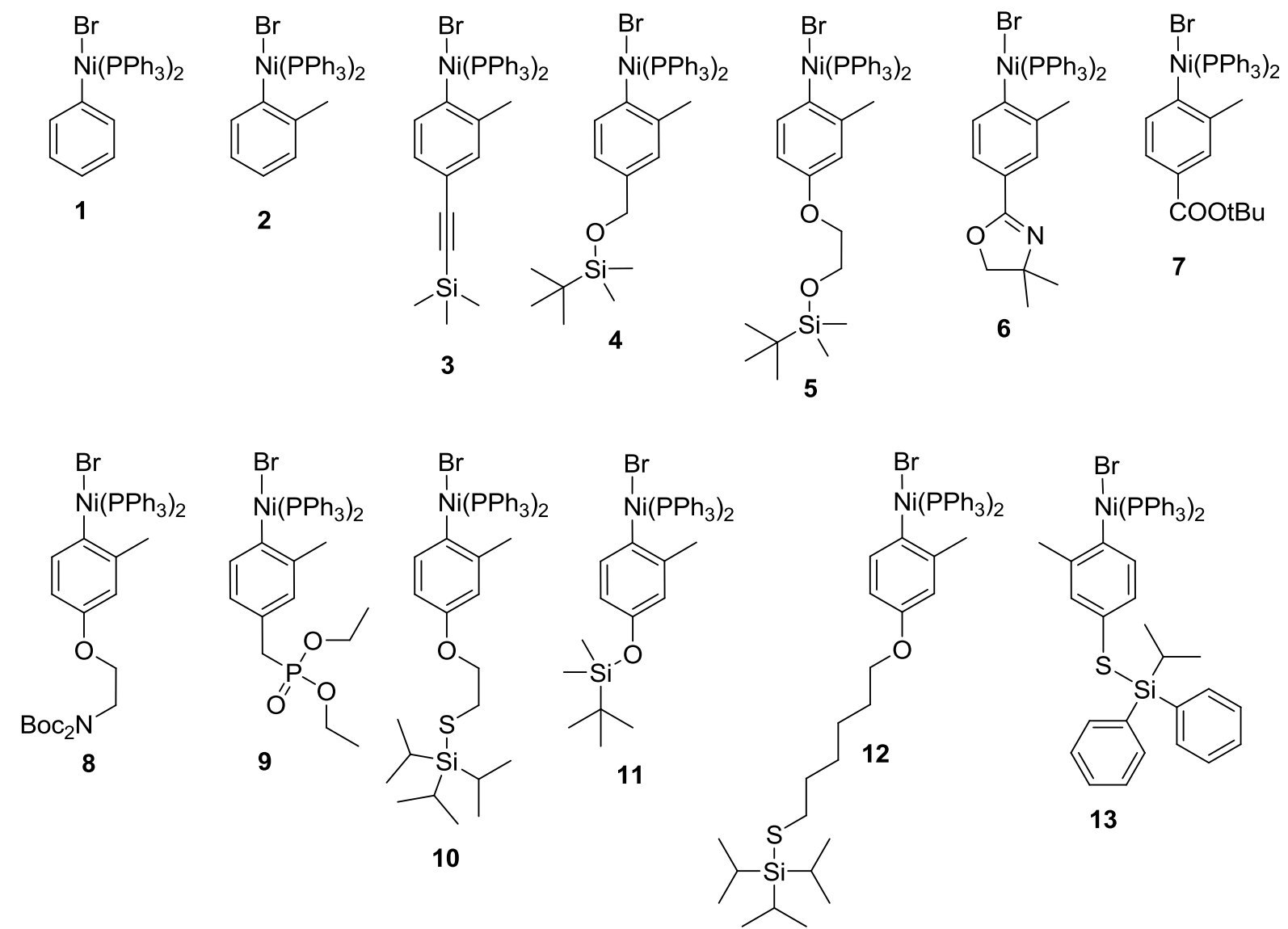

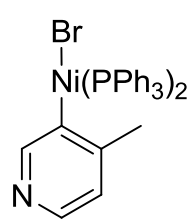

14

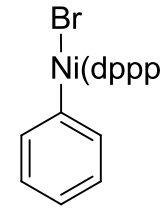

15
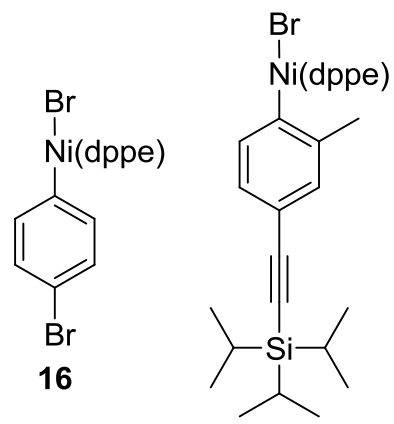

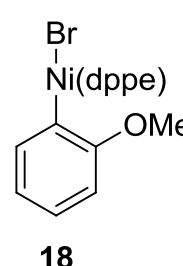

18
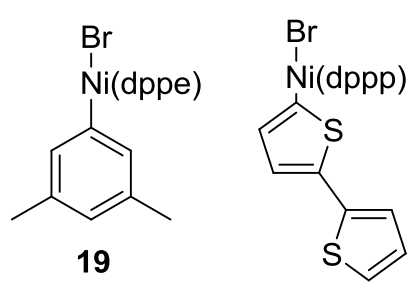

20

17

Scheme 4: Purified external Ni-initiators used in KCTP.

All previously mentioned external initiators were used in KCTP, but also for other CTPs external initiators were utilized. Initiator $\mathbf{1 5}$ was for example also used in the polymerization of the anion-radical complex of thiophene naphtalene diimide oligomers ${ }^{56}$ and in the synthesis of PPE using a Sonogashira reaction ${ }^{9}$. For Suzuki-Miyaura CTP different $(\mathrm{Ar}) \mathrm{Pd}\left(\mathrm{P}^{\mathrm{t}} \mathrm{Bu}_{3}\right) \mathrm{Br}$ were designed and used in purified form for the synthesis of poly(fluorene) (PF) ${ }^{8,57-63}$, $\mathrm{P} \mathrm{AT}^{58,64-66}, \mathrm{PPP}^{57,62,66}$, poly(3,6-phenanthrene) $(\mathrm{PPhen})^{67}$, n-type fluorene copolymers ${ }^{68}$ and 
poly(pyridine-3,6-diyl) (PPyr ${ }^{69}$. However, the synthesis of the last polymer was not controlled due to the presence of disproportionation reactions. The external initiators were obtained by combining $\operatorname{Pd}\left(\mathrm{P}^{t} \mathrm{Bu}_{3}\right)_{2}$ with the desired aryl bromide. An overview of those initiators can be found in Scheme 5 .

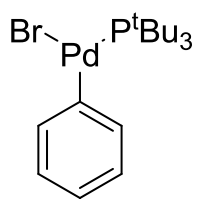

21<smiles>CCP(Br)c1ccccc1C</smiles>

22

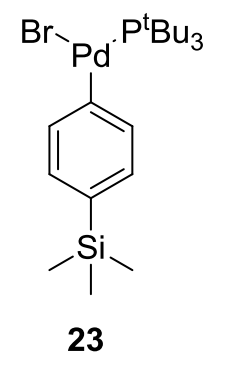

23

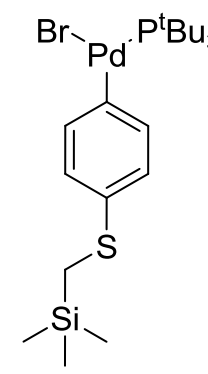

24

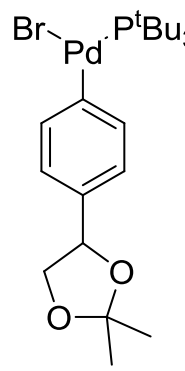

25

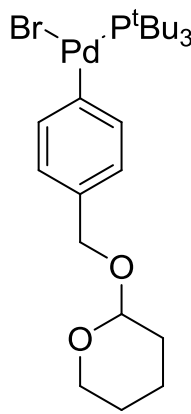

26

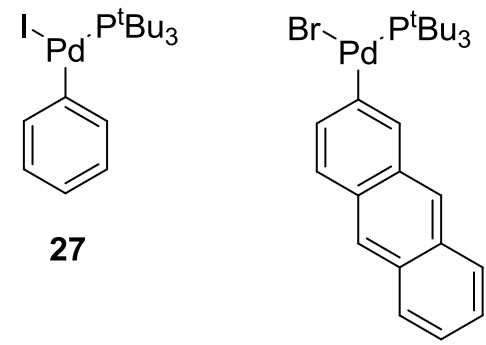

28

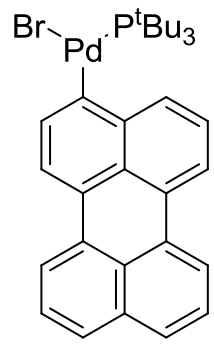

29
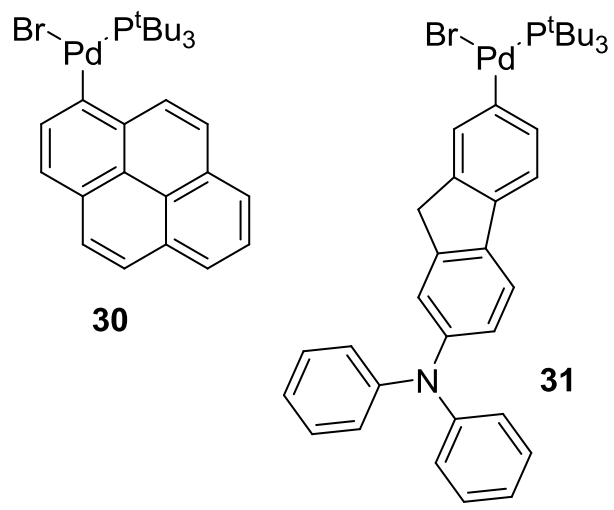

Scheme 5: Purified external Pd-initiators.

In contrast to external nickel initiators, in situ synthesized external palladium initiators can outperform the purified ones in Suzuki-Miyaura CTP. When for example $\operatorname{Pd}_{2}\left(\mathrm{dba}_{3}\right)(\mathrm{dba}=$ dibenzylideneacetone), ${ }^{\mathrm{t}} \mathrm{Bu} 3 \mathrm{P}$ and an aryl halide are used in a combined catalyst system, $\operatorname{ArPd}\left({ }^{\mathrm{t}} \mathrm{Bu}_{3} \mathrm{P}\right) \mathrm{X}$ is formed in situ. The polymerization with this initiator proceeds in a controlled way and narrow dispersities were obtained. ${ }^{62}$ It was also found that an additional amount of ${ }^{t} \mathrm{Bu}_{3} \mathrm{P}$ yielded polymers with a lower dispersity, probably due to the formation of the more stable $\mathrm{Pd}\left({ }^{\mathrm{t}} \mathrm{Bu}_{3} \mathrm{P}\right)_{\mathrm{n}}(\mathrm{n} \geq 2)$, and that $\mathrm{ArX}$ with various substituents in the para-position (i.e. $\mathrm{Cl}, \mathrm{Br}, \mathrm{F}, \mathrm{NO}_{2}, \mathrm{CN}, \mathrm{COPh}, \mathrm{CO}_{2} \mathrm{Et}, \mathrm{OMe}$ and $\mathrm{HOCH}_{2}$ ) can be used. ${ }^{61}$ 
Besides external initiators with one active site, also initiators with $2^{70}, 3^{70}, 4^{71}$ or $6^{72}$ active sides were prepared. These types of initiators give rise to special topologies, which will be discussed in 4. Advanced Topologies.

\subsubsection{Grafting from surfaces and nanoparticles}

For their implementation in optoelectronic devices, the topology and molecular organization of the used polymers is of great importance, since this has a large influence on the properties. ${ }^{73-76}$ Therefore, well-defined structures are desirable and this can be achieved by attaching the polymer chains covalently to surfaces. In this way new structures and better interactions between the polymers and the other parts of the device can be obtained. One way to achieve this goal is the use of initiators that are covalently bound to metal or glass surfaces. When, for example, aryl bromides are present on the surface, the $\mathrm{C}-\mathrm{Br}$ bond can be used to oxidatively insert $\mathrm{Ni}(0)$ or $\mathrm{Pd}(0)$. In this grafting from method a low dispersity and high grafting density can be obtained, but only for chain-growth polymerizations, like $\mathrm{KCTP}^{28,77}$ or Sonogashira polymerizations ${ }^{78}$. With Ni-catalysts, a high grafting density can sometimes give rise to disproportionation reactions and the surface coverage is not always equally divided. For palladium catalysts this is however less of a problem. ${ }^{79,80}$

The first surface initiated polymerization was performed by the group of Kiriy. Photocrosslinked poly(4-bromostyrene) films were reacted with $\mathrm{Ni}\left(\mathrm{PPh}_{3}\right)_{4}$ to obtain a macroinitiator used for the KCTP of P3AT. ${ }^{28}$ Poly(4-vinylpyridine)- $b$-poly(4-iodostyrene), adhered to a variety of polar substrates including silica particles, glass or metal oxide surfaces by the polar P4VP block, were used in the same way. ${ }^{81}$ Crosslinked poly(4-bromostyrene) was after reaction with $\mathrm{Pd}\left(\mathrm{P}^{\mathrm{t}} \mathrm{Bu}_{3}\right)_{2}$ also used as external initiator for the Suzuki-Miyaura polymerization of fluorene monomers. ${ }^{82}$ Later on, the group of Locklin succeeded in the KCTP of PPP and P3AT from gold surfaces. ${ }^{77,83,84}$ To synthesize the initiator, $\mathrm{Ni}(\mathrm{COD})_{2}$ and $\mathrm{PPh}_{3}$ were combined, followed by the oxidative addition of the formed $\mathrm{Ni}(0)$ species into the 
$\mathrm{C}-\mathrm{Br}$ bond of a thienyl bromide that was bound to the gold surface. Based on the better performance of bidentate ligands in KCTP, the group of Kiriy made a new initiator bound to silica nanoparticles. First, Ni(bipy)Et $t_{2}$ was combined with an immobilized aryl halide, followed by a ligand exchange with dppp or dppe. ${ }^{35,85-87}$ Islam et al. adjusted the procedure using a thiophene halide instead of an aryl halide ${ }^{88}$ and Sontag et al. used the same procedure, but employed gold surfaces instead of silica nanoparticles ${ }^{79}$. Also $\mathrm{TiO}_{2}$-nanoparticles were used to initiate the KCTP of thiophene monomers. ${ }^{89}$ Due to the ligand exchange a more stable catalyst system is obtained, but unfortunately it can also cause loss of surface initiator coverage. Therefore, a catalyst system with a bidentate ligand, but synthesized without a ligand exchange step, was designed. First, the electrochemical reduction of arene diazonium salts generates a bromobenzene monolayer on a gold surface. In a second step $\mathrm{Ni}(\mathrm{dppp}) \mathrm{Cl}_{2}$ is electrochemically reduced to $\mathrm{Ni}(\mathrm{dppp})$, which can then insert into the $\mathrm{C}-\mathrm{Br}$ bond of the bromobenzene. ${ }^{90}$ Recently, Youm et al. reported a second method without a ligand exchange. First a thiophene-based external initiator with a triethoxysilyl functionality was synthesized, followed by the immobilization of this compound on silica surfaces. ${ }^{91}$ Also palladium catalysts can be bound to surfaces. This was for example done by the group of Locklin. They synthesized an indium tin oxide surface functionalized with a (4-bromobenzyl)phosphonic acid monolayer and let it react with $\operatorname{Pd}\left(\mathrm{P}^{t} \mathrm{Bu}_{3}\right)_{2}$. This surface bounded initiator was then used for the KCTP of thiophene monomers. ${ }^{80}$ In the group of Bielawski, a surface bounded initiator for the Sonogashira synthesis of poly(para-phenylene ethynylene) (PPE) was synthesized by the addition of $\mathrm{Pd}\left(\mathrm{P}^{t} \mathrm{Bu}_{3}\right)_{2}$ to an aryl bromide which was bound to silica nanoparticles. ${ }^{78}$ Besides the initiation from surfaces and nanoparticles, it is also possible to incorporate initiating moieties into polymers in solution. It is, for example, possible to use the grafting from technique starting from (non-)conjugated polymer backbones, which will be described in 
more detail in section 4. Advanced Topologies, or to use (non-)conjugated polymers that can be converted into macroinitiators. ${ }^{92}$

\subsection{Propagation}

After the initial transmetalation, reductive elimination and oxidative addition during the initiation, the same catalytic cycle is repeated during the propagation (Scheme 1). To obtain control over the polymerization it is of utmost importance that the catalyst remains associated to the polymer backbone between the reductive elimination and oxidative addition. In this way, only intramolecular oxidative addition is possible and transfer and termination reactions are suppressed.

\subsubsection{Kumada catalyst transfer polymerization (KCTP)}

KCTP, originally called Grignard metathesis (GRIM) polymerization, is the most extensively investigated CTP and is based on the Kumada coupling of monomers bearing a halide and a magnesium halide function. ${ }^{93}$ Those organomagnesium compounds are very sensitive to moisture and are therefore generated in situ starting from the precursor monomer. Often a small excess of precursor monomer is used, since metalation reagents, present after the incomplete formation of the monomer, can act as termination reagents during the polymerization, while the presence of the precursor monomer has no influence. Nickel as well as palladium catalysts can be used in KCTP, but overall Ni-catalysts give rise to a higher degree of control over the polymerization. Therefore Ni-catalysts are more used and studied, although also KCTP with $\mathrm{Pd}\left(\mathrm{PPh}_{3}\right)_{4}$ was reported. ${ }^{80,94}$ The main problem with this Pd-catalyst is the weaker association with the polymer backbone compared to their Ni counterparts. To overcome this problem, more electron donating ligands, i.e. NHC ( $N$-heterocyclic carbene) ligands, were used and more control over the polymerization was obtained. ${ }^{95}$

The most studied monomer used in KCTP is thiophene. ${ }^{3,4}$ This is a rather electron rich monomer, resulting in a good association between the polymer backbone and the catalyst and, 
hence, control over the polymerization. Different thiophene monomers were used, but most of them are substituted in the 3-position, introducing asymmetry. In this way head-to-tail, tail-totail and head-to-head couplings are possible (Scheme 6), resulting in regioregular or regioirregular P3AT. Which of the couplings will be formed depends on the used precursor monomer and catalyst. When 3-substituted 2,5-dibromothiophene is used, the Grignard metathesis reaction results in the formation of two isomers and their ratio depends on the substituent of the thiophene and the Grignard reagent used. ${ }^{96}$ Both isomers can be polymerized when a Pd-catalyst is used and regio-irregular P3AT is obtained. ${ }^{94}$ However, when a Ni-catalyst with bulky ligands is used, only the 2-bromo-5-chloromagnesio-3hexylthiophene can be built in, since head-to-head couplings are hardly promoted due to the steric crowding around the Ni center, and regioregular PT is obtained. The regioregularity can still be decreased using less sterically demanding ligands or by the addition of $\mathrm{LiCl}$. $\mathrm{LiCl}$ breaks up the aggregates of the Grignard reagent and forms ate-complexes, which can increase the propagation rate and facilitate the formation of head-to-head couplings. ${ }^{97,98}$ When sterically demanding ligands in combination with $\mathrm{LiCl}$ are used, both isomers will be incorporated in the polymer, but first the 2-bromo-5-chloromagnesio-3-hexylthiophene polymerizes, followed by the 5-bromo-2-chloromagnesio-3-hexylthiophene, resulting in the formation of a block copolymer. To overcome the problem of regioregularity, other thiophenes were used as precursor monomer. 3-substituted 2-bromo-5-iodothiophene was used in the selective Grignard metathesis method, which results in the substitution of only the iodine. ${ }^{99}$ Finally, also 2-bromo-3-alkylthiophene was used in two different methods. The first one is the McCullough method in which subsequently LDA (lithium diisopropylamide) and $\mathrm{MgBr}_{2}$ are added. ${ }^{100,101}$ The second one is based on the addition of the Knochel-Hauser base, TMPMgCl$\cdot \mathrm{LiCl}$ (chloromagnesium 2,2,6,6-tetramethylpiperidine lithium chloride salt). ${ }^{102,103}$ Other polymers that resemble poly(thiophene)s were also synthesized in a controlled way 
with KCTP, namely P3ASe ${ }^{104,105}$, poly(3-alkyltellurophene)s $(\mathrm{P} 3 \mathrm{ATe})^{106}$ poly(pyrrole)s $(\mathrm{PP})^{107-110}, \quad$ poly(thiazole)s $\quad(\mathrm{PTZ})^{54,111}, \quad$ poly(dithienosilole)s $\quad(\mathrm{PDTS})[107,108]$ and poly(cyclopentadithiophene)s (PCPDT) ${ }^{114}$ (Scheme 7).<smiles>[R]c1cc(N2[C]CCC[CH]2)cc([R])c1Br</smiles>

tail-to-tail<smiles>[R]c1cc(N2[C]CCC[CH]2)c([R])s1</smiles>

head-to-tail<smiles>[R]c1cc(Br)sc1C1CCC[C]N1c1sc(Br)cc1[R]</smiles>

head-to-head

Scheme 6: Illustration of all possible couplings for P3AT. 

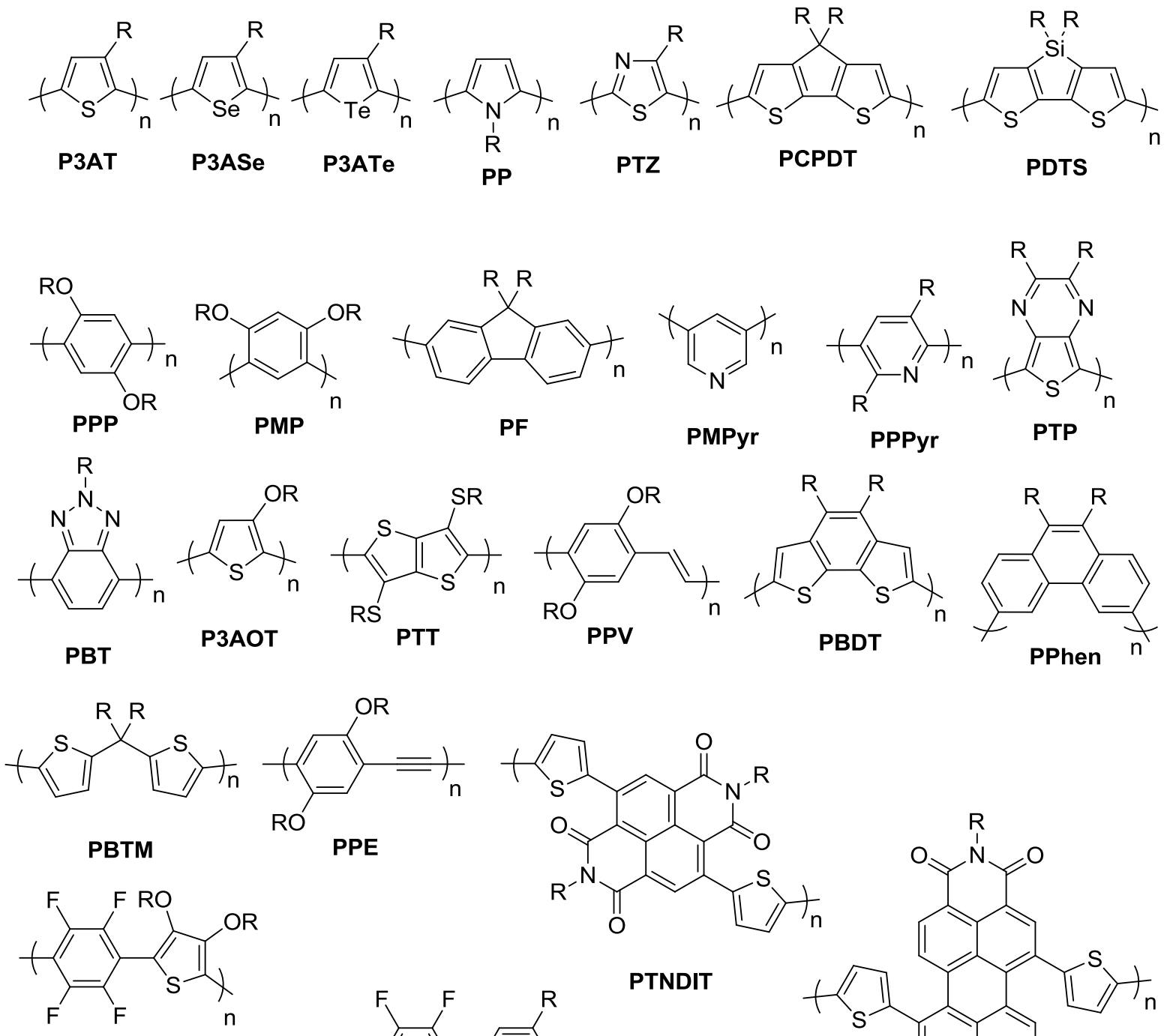

P(PP-alt-T)<smiles>[R]N1C(=O)c2cc(-c3ccc(C(C)(C)C)s3)c3c4c(cc(-c5ccc(C(C)(C)C)s5)c(c24)C1=O)C(=O)N([R])C3=O</smiles>

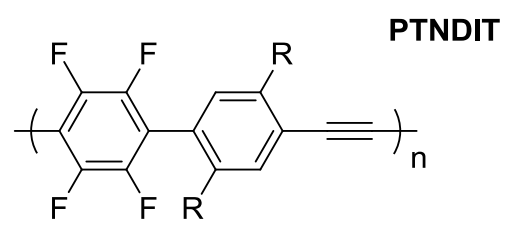

P(PP-alt-PE)

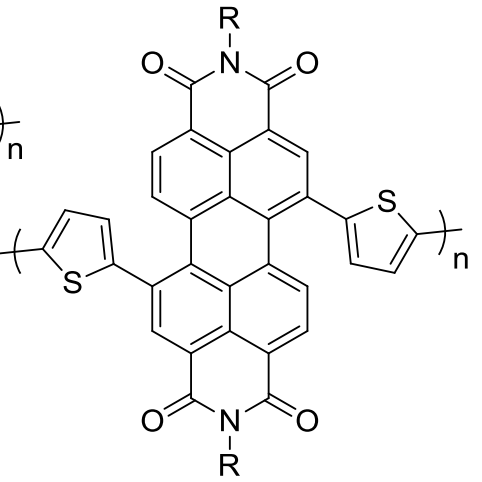

PTPDIT

Scheme 7: Illustration of all polymers synthesized by CTP.

In addition to heteroaromatic monomers, also all-carbon monomers were successfully used in KCTP. It is, however, more difficult to obtain control over the polymerization since those monomers are less electron rich than most of their heteroaromatic counterparts. The phenylenes used are therefore often equipped with two alkoxy side chains to increase the electron density of the aromatic ring and the strength of the complexation with the catalyst. Since the monomer is symmetric, 1,4-dibromo-2,5-dialkoxybenzene ${ }^{115}$ or 1,4-diiodo-2,5- 
dialkoxybenzene ${ }^{83}$ can be used as precursor monomer without any consequences for the regioregularity. Besides PPPs, also poly(meta-phenylene)s (PMP) were made by KCTP. ${ }^{116}$

For fluorene monomers, not only the lower electron density is a problem to obtain control over the polymerization, but also the length of the monomer. The catalyst needs to stay complexed to the polymer backbone during the time between reductive elimination and subsequent oxidative addition and due to the larger dimensions of fluorene, this time will be longer than for phenylene monomers. Since regioregularity is no issue for PFs, both 2,7dibromofluorene and 7-bromo-2-iodofluorene can be used as precursor monomer. When Nicatalysts are used with the usual bidentate phosphine ligands (dppp or dppe), a non-controlled chain-growth polymerization is obtained. ${ }^{52,117,118}$ However, when nickel acetylacetonate $\left(\mathrm{Ni}(\mathrm{acac})_{2}\right)$ and dppp are used in a combined catalyst system, control over the polymerization is achieved. ${ }^{119}$

Electron deficient monomers were also used in an attempt to polymerize them in a controlled manner using KCTP. However, due to their lower electron density this was not always successful. Poly(pyridine-3,5-diyl) (PMPyr) can be synthesized in a controlled way with $\mathrm{Ni}(\mathrm{dppp}) \mathrm{Cl}_{2}{ }^{12,120}$, but for the regioisomer poly(pyridine-3,6-diyl) (PPPyr) this is not the case due to the presence of disproportionation reactions ${ }^{69}$. The synthesis of poly(benzotriazole) (PBT) was found to be controlled only when Ni(II) diamine catalysts were used. ${ }^{121,122}$ For $\mathrm{PTP}^{42,123}$ no catalyst system was found up to now that allows a controlled synthesis.

Besides an inadequate association between the catalyst and the polymer backbone, also a more difficult oxidative addition can result in a dissociation that becomes competitive with the oxidative addition. This results in decomplexation of the catalyst and termination of the polymerization and is, for example, the case for P3OATs. ${ }^{50}$ Besides dissociation of the catalyst, also a too strong complexation can inhibit the polymerization. The polymerization of thienothiophene via KCTP is for example not possible due to the formation of a very stable 
$\mathrm{Ni}(0)$-complex. ${ }^{7}$ Also during an attempt to polymerize $p$-phenylene vinylene, it was found that the association of the $\mathrm{Ni}(0)$-catalyst and the diene was too strong to allow polymerization $^{124}$. Later, the polymerization of benzodithiophene was attempted, but the presence of the 'non-aromatic' double bond introduced transfer reactions, leading to loss of control over the polymerization. ${ }^{125}$ Normally the double bond is considered as a part of the aromatic system, but due to the almost equal energy of the total aromatic system and the system with the double bond not being part of the aromatic system, this double bond can behave as a 'normal' double bond. A final problem that was encountered for KCTP was the incomplete conversion of the Grignard metathesis reaction necessary to convert the precursor monomer, leaving unreacted metalation reagents in the reaction mixture, which can act as termination reagents. This was the case in the synthesis of poly(3,6-phenanthrene) (PPhen). ${ }^{67}$ Also one non-conjugated polymer was synthesized with KCTP, namely poly(bithienylmethylene) (PBTM). ${ }^{126}$ The polymer backbone is not conjugated, but does contain an aromatic repeating unit.

In order to obtain a universal catalyst system which can be used for the KCTP of different monomers, it is of utmost importance to understand the influence of the ligand on the polymerization. In the group of McNeil different rate and spectroscopic studies on the KCTP of 4-bromo-1-chloromagnesio-2,5-di(hexyloxy)phenylene and 2-bromo-5-chloromagnesio-3hexylthiophene were conducted and it was found that the steric and electronic properties of the ligand used have an important influence on the polymerization mechanism. When, for example, $\mathrm{Ni}(\mathrm{dppe}) \mathrm{Cl}_{2}$ is used, the reductive elimination is the rate determining step $^{127}$, while for $\mathrm{Ni}(\mathrm{dppp}) \mathrm{Cl}_{2}$ the rate determining step is the transmetalation ${ }^{128}$. This difference in rate determining step also explains why the addition of $\mathrm{LiCl}$ has no influence on the polymerization with $\mathrm{Ni}(\mathrm{dppe}) \mathrm{Cl}_{2}$, but does increase the polymerization rate when $\mathrm{Ni}(\mathrm{dppp}) \mathrm{Cl}_{2}$ is used. This shows that the bite angle of the ligand can have a large influence on the 
polymerization mechanism. Later on, three other bidentate phosphine ligands with different steric crowding were tested. ${ }^{110}$ The first one was $\mathrm{Ni}($ depe $) \mathrm{Cl}_{2}$ (depe = bis(diethylphosphino)ethane) and it was found that the reductive elimination was the rate determining step. The polymers showed a low dispersity, but also low molar mass tailing due to the slower initiation compared to the propagation. With $\mathrm{Ni}(\mathrm{dcpe}) \mathrm{Cl}_{2}$ (dcpe = bis(dicyclohexylphosphino)ethane) the transmetalation is the rate determining step. This is explained by the increased steric crowding around the Ni center, accelerating the reductive elimination and slowing down the transmetalation. With this catalyst only oligomers could be prepared, due to the reduced association of the $\mathrm{Ni}(0)$-species to the polymer backbone. For the third catalyst, $\mathrm{Ni}(\mathrm{dmpe}) \mathrm{Cl}_{2}(\mathrm{dmpe}=$ bis(dimethylphosphino)ethane $)$, the reductive elimination was again the rate determining step, but due to the small amount of steric crowding, the catalyst degraded quickly. The last factor that was investigated is the electronic properties of the ligands. It was found that the most electron donating ligand resulted in the lowest dispersity. ${ }^{129}$ This can be explained by the larger stabilization of the Ni(0)-polymer $\pi$-complex and acceleration of the oxidative addition, both resulting in the suppression of competing reaction pathways, like chain transfer and termination. Also a relative acceleration of the precatalyst initiation compared to the propagation was obtained.

In 2010 the group of Kiriy found that the catalyst can 'walk' over the polymer backbone during the polymerization, causing bidirectional growth of the polymer chain. This process is referred to as 'random catalyst walking' and was discovered by the use of external initiator 16. None of the polymers obtained had the phenyl ring at the beginning of the chain, leading to the conclusion that the catalyst was able to walk over the entire polymer backbone after reductive elimination in order to reinsert in the $\mathrm{C}$-Br bond at the other chain end. ${ }^{51}$ Complementary evidence was delivered by our group in a study about the position of the tailto-tail dimer, formed during the initiation with Ni-salts, in the P3AT polymerization. It was 
found that this tail-to-tail dimer is not always located at the beginning of the polymer chain, indicating that 'random catalyst walking' occurs. ${ }^{43}$ In 2012, Kohn et al. validated this 'random catalyst walking' using various calorimetric and scattering experiments. ${ }^{130}$ The consequences of this 'random catalyst walking' for the synthesis of homopolymers are limited, but for block copolymers this can be detrimental. When $\mathrm{AB}$ block copolymers are desired, it is possible that BAB-block copolymers are obtained and for endcapping, the 'random catalyst walking' can give rise to dicapping. A more elaborated discussion of the consequences and solutions will be given in sections 2.2.8. All-conjugated block copolymers and 2.3. Termination and endcapping in CTP.

\subsubsection{Negishi catalyst transfer polymerization (NCTP)}

When monomers with an organozinc and a halide function are synthesized, NCTP can be used to obtain the corresponding polymers (Scheme 8). Nickel as well as palladium catalysts have been used for this purpose. In the group of McCullough the first NCTP was conducted with 2bromo-5-chlorozinc-3-hexylthiophene. The monomer was synthesized in situ from 2-bromo3-hexylthiophene, by the sequential addition of LDA and $\mathrm{ZnCl}_{2 \cdot}{ }^{4,131}$ Later on, Higashihara et al. used a new synthetic route to obtain the necessary monomer, based on the reaction between 2-bromo-5-iodo-3-hexylthiophene and ${ }^{t} \mathrm{Bu}_{4} \mathrm{ZnLi}_{2}$. The polymerization resulted in materials with low dispersities and the monomer synthesis was not as susceptible to moisture or protic impurities as was the case for the previously mentioned synthetic method for organozinc monomers. ${ }^{132,133}$ All polymerizations of thiophene monomers based on NCTP were catalyzed by Ni-catalysts. A Pd-catalyst, $\mathrm{Pd} / \mathrm{P}^{t} \mathrm{Bu}_{3}$, has been used for the synthesis of PFs. ${ }^{134}$ 


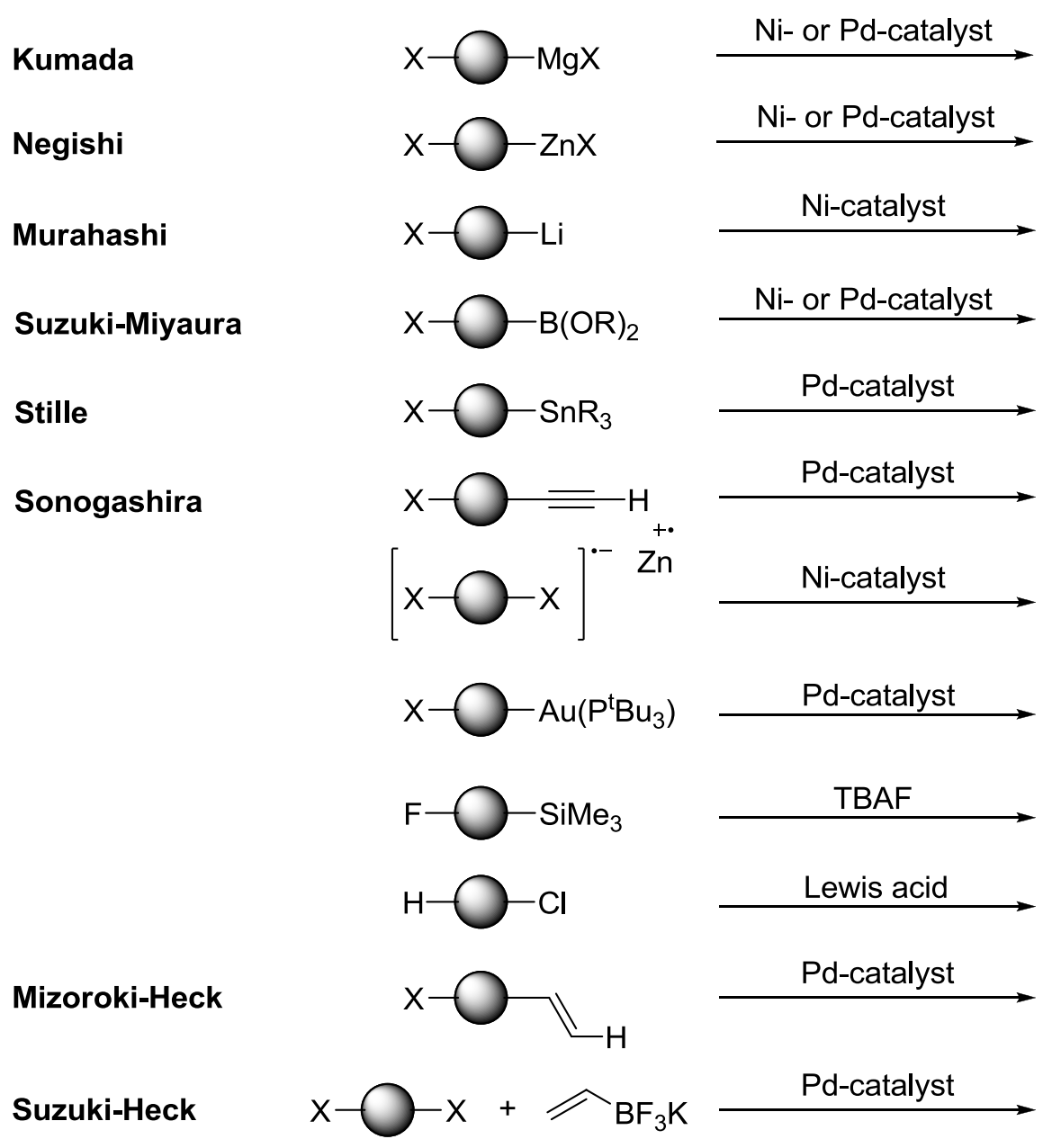

Scheme 8: Illustration of all monomers and relevant polymerization reactions that can follow catalyst transfer mechanisms.

\subsubsection{Murahashi catalyst transfer polymerization}

The next type of organometallic monomers is the one with an organolithium and a halide function, used in Murahashi catalyst transfer polymerizations. Using Ni(NHC)-catalysts, P3ATs as well as PPPs were synthesized. The monomers were obtained via deprotonation or lithium-halogen exchange of the precursor monomers. ${ }^{11}$ For the lithium-halogen exchange of the thiophene precursor monomer only the chlorinated monomer can be used if regio-regular P3ATs are desired. This type of CTP is, however, not often used. 


\subsubsection{Suzuki-Miyaura catalyst transfer polymerization (SCTP)}

Besides organometallic monomers that are prepared in situ, also monomers that allow purification can be used in CTPs. For example, monomers used for SCTP, containing a boronic acid or ester and a halide functionality. Those monomers are air stable, can be purified with column chromatography, allow mild reaction conditions and tolerate a large variety of functional groups. Deboronation and dehalogenation of the monomer can however occur $^{135}$ and optimal reaction conditions depend on the monomer used ${ }^{136-139}$. Mostly Pdcatalysts are used in SCTP and small amounts of water are added to favor the intramolecular transfer of this catalyst. ${ }^{65}$ Yokoyama et al. were the first to use Suzuki-Miyaura couplings to obtain a chain-growth polymerization. PF with a low dispersity was obtained when $(\mathrm{Ph}) \mathrm{Pd}\left(\mathrm{P}^{\mathrm{t}} \mathrm{Bu}_{3}\right) \mathrm{Br}$ was used as catalyst. ${ }^{8}$ Later on, also other catalytic systems were found to be successful for SCTP of PFs. ${ }^{61,62,140,141}$ Besides PFs also PPP ${ }^{57}$ and P3AT $65,141,142$ were synthesized in a controlled way with SCTP using Pd-catalysts. Recently, a controlled Nicatalyzed SCTP of poly(thiophene)s was performed. ${ }^{26}$

The polymerization of pyridine-3,6-diyl was not controlled due to the occurrence of disproportionation reactions, as it was also the case when KCTP was performed on this monomer. ${ }^{69}$ Also the synthesis of PPyr was not controlled due to the limited association of the catalyst to the polymer backbone. ${ }^{67}$ During an attempt to synthesize poly(p-phenylene vinylene) (PPV) the opposite problem was encountered. The $\mathrm{Pd}(0)$-species, which is formed after the reductive elimination, is trapped by the vinylene bond, inhibiting further propagation. ${ }^{124}$ Later on, it was found that ortho-substituents relative to the vinylene bond promote the intramolecular transfer, ensuring chain-growth. ${ }^{143}$

\subsubsection{Stille catalyst transfer polymerization}

Instead of incorporating boronic acids or boronic esters into the monomer, also organotin functionalities can be used. The main drawback of this method is the high toxicity of the 
organotin compounds necessary during the synthesis of the monomers, but a lot of functional groups can be incorporated into the monomer and no optimization of the base, organoboron moiety or the amount of water is required. ${ }^{65}$ As well for $\mathrm{PPE}^{9}$ as for $\mathrm{P} \mathrm{AT}^{10}$ controlled chaingrowth polymerizations were obtained via Stille catalyst transfer polymerization.

\subsubsection{Sonogashira catalyst transfer polymerization}

Besides Stille couplings, also Sonogashira couplings were investigated to obtain control over the polymerization of $p$-phenylene ethynylene. It was found that PPE with low dispersities could be synthesized with $(\mathrm{Ph}) \mathrm{Pd}\left(\mathrm{P}^{t} \mathrm{Bu} 3\right) \mathrm{Br}, \mathrm{CuI}$ and $\mathrm{PPh}_{3}$ in a combined catalyst system, but unfortunately the monomer conversion was rather low. ${ }^{9}$

\subsubsection{Miscellaneous}

In 2011, the group of Kiriy found a new method for the controlled polymerization of electron deficient brominated thiophene-naphthalene diimide oligomers. ${ }^{56}$ When the precursor monomer is combined with Rieke Zinc ${ }^{144}$, not the expected organozinc compound is obtained, but an anion-radical complex is formed instead. With $\mathrm{Ni}(\mathrm{dppe}) \mathrm{Br}_{2}$ or $(\mathrm{Ph}) \mathrm{Ni}(\mathrm{dppe}) \mathrm{Br}$ a controlled chain-growth polymerization is obtained and ${ }^{31} \mathrm{P}$ NMR studies showed that also in this type of polymerization the complexation between $\mathrm{Ni}(0)$ and the polymer backbone is the key to success. ${ }^{6}$ Despite the control over the polymerization, high dispersities are obtained. This is explained by the uncontrolled initiation, involving a two-electron transfer process from the anion-radical monomer to the Ni-catalyst. The generated $\mathrm{Ni}(0)$-complex can then insert into the $\mathrm{C}-\mathrm{Br}$ bond of the monomer and start the polymerization. It was also found that the 'random catalyst walking' was more extensively than for other monomers, probably due to the better shieling of the polarizing effect of the $\mathrm{C}-\mathrm{Br}$ bond by the electron deficient naphthalene-diimide group present in the monomer. The success of this type of polymerization led to the extension to $\mathrm{Pd}$-catalysts ${ }^{145}$ and perylene diimide-based monomers ${ }^{146}$. 
Recently, Suraru et al. reported the controlled polymerization of aurylated alkylthiophene monomers catalyzed by Pd-PEPPSI- ${ }^{i} P r .{ }^{147}$ The monomers were synthesized by the addition of $\left({ }^{\mathrm{t}} \mathrm{Bu} 3 \mathrm{P}\right) \mathrm{AuCl}$ to 2-bromo-3-alkylthiophene.

All previously mentioned controlled catalyst transfer polymerizations were based on the association of the $\mathrm{Ni}$ - or Pd-catalyst to the polymer backbone. It is however also possible that a fluoride anion fulfills this task. Sanji et al. found that 2-perfluoroaryl-5trimethylsilylthiophenes can be polymerized in a controlled way without the addition of a transition metal, but with a catalytic amount of TBAF (tetra- $n$-butylammonium fluoride), yielding $\operatorname{poly}(p$-tetrafluorophenylene-alt-thienylene $)(\mathrm{P}(\mathrm{PP}-$ alt $-\mathrm{T})) .{ }^{148} \mathrm{~A}$ pentacoordinated fluorosilicate was found to be the key intermediate and polymers with a controlled molar mass and low dispersity were obtained. Later on, this polymerization mechanism was also used for the controlled synthesis of $\operatorname{poly}(p$-tetrafluorophenylene-alt-phenylene ethynylene) $(\mathrm{P}(\mathrm{PP}-$ alt $-\mathrm{PE})){ }^{149}$

Also in the cationic chain-growth polymerization of 2-chloroalkylenedioxythiophene monomers, no transition metal catalyst is utilized. ${ }^{150}$ Instead, a Lewis acid in catalytic amounts is used. After the polymerization, a non-conjugated polymer is obtained, but during the workup with methanol and hydrazine hydrate, $\mathrm{HCl}$ is eliminated and the conjugation is restored.

A last polymerization method is based on Mizoroki-Heck couplings. Nojima et al. tried to synthesize PPV is this way, but unfortunately no controlled polymerization was obtained due insufficient association of the catalyst to the polymer backbone. ${ }^{151}$ The combination of Heck with Suzuki-Miyaura couplings to obtain poly(fluorenylene-vinylenes) was also attempted. The polymerization showed chain-growth characteristics in the first stage, but after the consumption of the fluorene monomer, the polymerization proceeded in a step-growth manner. ${ }^{152}$ 


\subsubsection{All-conjugated block copolymers}

In order to improve existing applications and create new opportunities, it is important to combine materials with different properties (e.g. mechanical properties, thermal stability, processability and hydrophobicity). Block copolymers form an attractive group of materials to accomplish this goal: their properties cannot be achieved with blends of their homopolymers. ${ }^{153,154}$ Multiple approaches are available for the synthesis of conjugated block copolymers, e.g. the grafting to method ${ }^{44,155}$, the end-functional polymer copolymerization method $^{154,156}$ and the grafting from method ${ }^{157,158}$. However, the most desired approach is sequential monomer addition. In contrast to the other mentioned methods, the synthesis consists of a direct one-pot reaction in which the second monomer is added after complete consumption of the first one. In this section, the synthesis of block copolymers through sequential monomer addition will be discussed.

\section{All-conjugated block copolymers with the same aromatic moieties}

When different conjugated systems are combined in one polymer, rod-rod block copolymers are obtained. The direct synthesis of these block copolymers via sequential monomer addition requires a controlled chain-growth polymerization. This implies not only the use of a catalytic system that is able to polymerize both monomers, but also under similar polymerization conditions. CTP is without doubt the most used polymerization technique for sequential monomer addition. This method results in well-defined block copolymers with a controlled molar mass and low dispersity.

The first block copolymers synthesized in this way consisted of the same aromatic moieties, but with different side chains. Already large assortments of side chains were combined. The research group of McCullough was the first to combine 3-alkylthiophene monomers with different side chain lengths. Not only diblock copolymers were formed, but even triblock copolymers were prepared. ${ }^{18} \mathrm{Wu}$ et al. investigated the influence of the difference in side 
chain length in poly(3-butylthiophene)-b-poly(3-octylthiophene) on the microphase separation ${ }^{159}$ and later, our group visualized this with $\mathrm{STM}^{160}$. Ge et al. further investigated the influence of the length of the side chain of block copoly(3-alkylthiophenes). They revealed that a difference exceeding two carbon atoms between the side chains of the blocks results in microphase separation. ${ }^{161}$ Similarly, Zhang et al. obtained a block copoly(3alkylthiophene) in which the second monomer is a branched 3-(2-ethylhexyl)thiophene. ${ }^{162}$ Again, microphase separation was induced. Our research group incorporated an achiral poly(3-hexylthiophene) block with a chiral poly(3-(3,7-dimethyloctyloxy)thiophene) block. ${ }^{163}$ The incorporation of chirality made it possible to investigate the influence of one conjugated block on the other one. Because of its success, this technique was further applied to investigate the supramolecular structure of conjugated block copolymers. ${ }^{164}$ Other examples are thiophenes containing alkylhalides ${ }^{165}$, fulleropyrrolidine ${ }^{166}$, lateral octylphenyl ${ }^{167}$, styryl $^{168}$, amine ${ }^{169}$, cyclohexyl ${ }^{170}$, acetate ${ }^{171}$, aliphatic hydrocarbon naphthalene diimide side chains $^{172}$, phenoxymethyl groups ${ }^{173}$ and other oxygen containing side chains ${ }^{174,175}$.

When a perfect AB-block copolymerization is desired, one needs to consider 'catalyst walking'. Once the polymerization has started, the catalyst is able to undergo intramolecular transfer to the active carbon-halogen bond at the other end of the growing chain, resulting in bidirectional growth (previously discussed in section 2.2.1. Kumada catalyst transfer polymerization (KCTP)). This is only possible when the catalyst remains associated with the conjugated $\pi$-backbone of the polymer between the reductive elimination and the oxidative addition. For the synthesis of homopolymers this is not really an issue, however when the second monomer is added for the synthesis of block copolymers, insertion at both ends can take place and a mixture of AB- and BAB- block copolymers is obtained (Scheme 9). The solution to this problem is found in the use of external initiators lacking an active carbonhalogen bond. ${ }^{43}$ If the catalyst binds stronger to the second monomer than to the first one, as 
can be the case if two electronically different monomers are used, it prefers to stay associated to the second block. In this way, catalyst walking is also prevented.

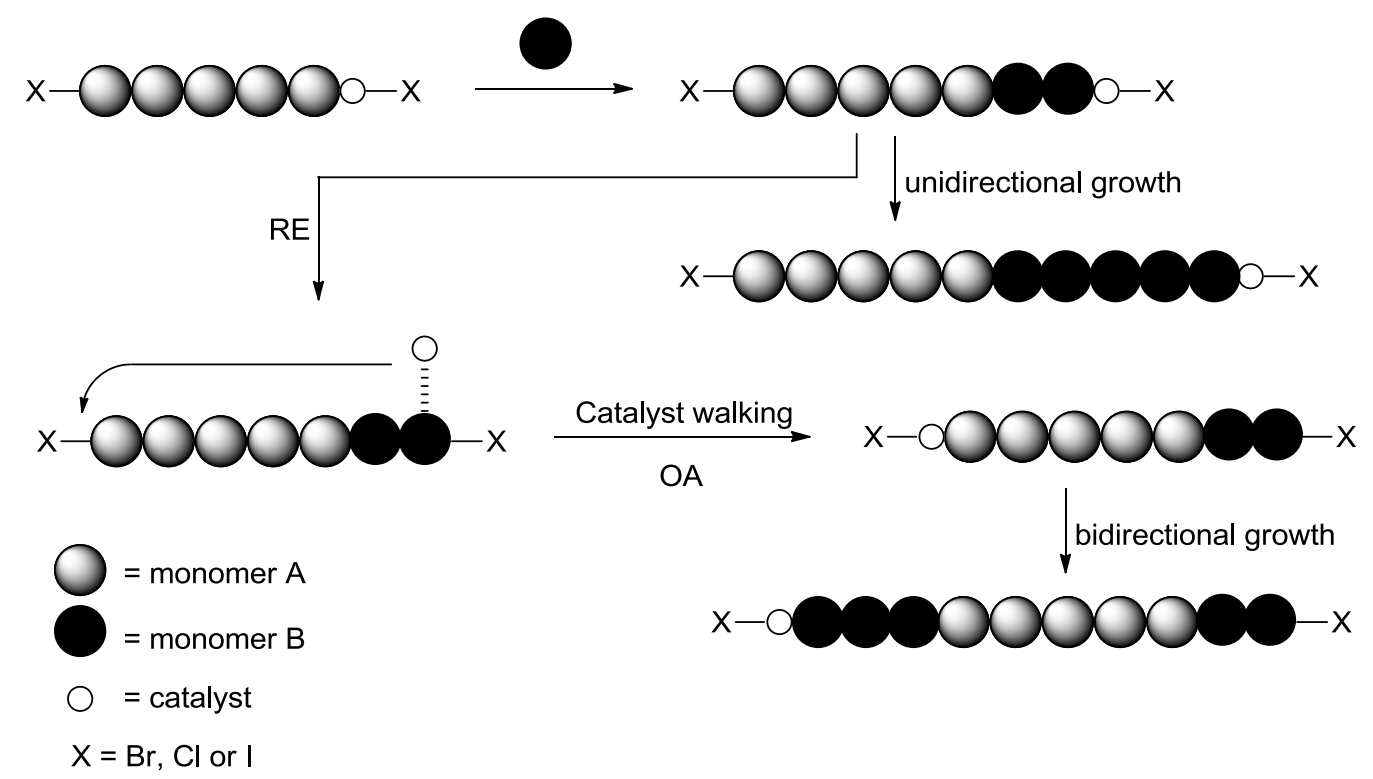

Scheme 9: Formation of AB- and BAB-block copolymers due to catalyst walking.

When the same aromatic moieties with similar side-chains are polymerized, the order of the blocks does not matter. However, when a combination of hydrophobic and hydrophilic side chains is used, the monomer with the hydrophobic side chain must be polymerized first. ${ }^{17-179}$ This sequential addition technique can also be extended to more advanced structures, e.g. the formation of triblock copolymers is possible by employing a bifunctional initiator. ${ }^{46}$ Additionally, block copolymers with a random copolymer as a second block were formed. ${ }^{166,180-182}$ Our research group prepared a block copolymer in which each block is a random copolymer. ${ }^{183}$

\section{All-conjugated block copolymers with different aromatic moieties}

Although an extensive number of all-conjugated block copolymers with the same aromatic moiety have appeared in literature, examples of block copolymers with different aromatic moieties are limited (Scheme 10). ${ }^{109}$ This can be attributed to two factors: the control of CTP relies on the complexation of the catalytic species to the $\pi$-conjugated system and monomers 
must undergo a controlled polymerization under the same reaction conditions. When electronically different monomers are used, it is more favorable for the catalyst to remain complexed with the most electron rich monomer or polymer chain. Therefore, the complexation between the catalytic species and the monomer must always increase during the polymerization or remain the same. If not, the catalyst will stay complexed to the first block, thereby ending the polymerization, as reported by the research group of Yokozawa. ${ }^{184}$ Other research groups confirmed this hypothesis by synthesizing block copolymers with electronically different monomers. ${ }^{185-187}$ Accordingly, $\mathrm{PF}^{119,187}, \mathrm{PPP}^{95,184,185}, \mathrm{PMP}^{116}$ and PDTS ${ }^{112}$ must be polymerized before P3AT, while PCPDT ${ }^{114}$ and $\mathrm{PTP}^{42}$ must be polymerized after P3AT. For the same reason also PPP must be polymerized before $\mathrm{PF}^{187}$ and $\mathrm{PP}^{109}$. When thiophenes with alkoxy ${ }^{163,164,176,188}$ and thioalkyl ${ }^{189}$ side chains are combined with 3alkylthiophenes, the monomer with the thioalkyl side chain must be polymerized first, followed by the monomer with the alkyl side chain and finally the monomer containing the alkoxy side chain. A second reason why alkoxy thiophenes must be polymerized last is its uncontrolled polymerization. In case the catalytic species complexes equally to electron rich and electron poor monomers, the order of addition is not important. The group of Seferos synthesized block copolymers of thiophene and benzotriazole without any effect of the monomer sequence. To accomplish this, they used a Ni(II) diimine catalyst, which polymerizes both monomers smoothly. ${ }^{122}$ Also block copolymers of P3AT and P3ASe can be copolymerized in both directions, due to the limited difference in electron density of the two monomers. ${ }^{105,190,191}$

Although KCTP is the most popular approach to obtain block copolymers, also other CTPs have been used. The research group of Higashihara was the first to accomplish a NCTP of poly(3-hexyltiophene) (P3HT) and poly(3-octadecylthiophene), resulting in well-defined block copolymers. ${ }^{192}$ Yokozawa polymerized PF and PPP using a SCTP. ${ }^{186}$ Also for this type 
of block copolymerization the order of addition was shown to be important. Later, SCTP was used to copolymerize PF and P3AT. ${ }^{141,142}$ The research group of Kiriy combined KCTP and NCTP, in which P3AT was polymerized via KCTP and PDTS via NCTP (Scheme 10). ${ }^{113}$

Also the $\mathrm{Pd}(\mathrm{RuPhos})$ polymerization, developed by our research group, can be used to produce block copolymers with different aromatic moieties. Using this method, triblock copolymers composed of three different aromatic moieties were produced for the first time. ${ }^{193}$ Since this polymerization mechanism does not rely on catalyst association but on deactivation of the monomers, this will be discussed in section 3. Monomer deactivation.

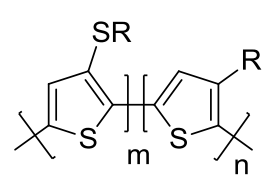

P3AST-b-P3AT

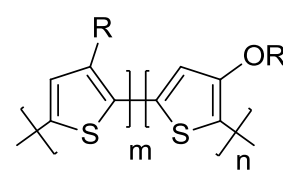

P3AT-b-P3AOT

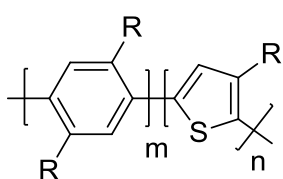

PPP-b-P3AT

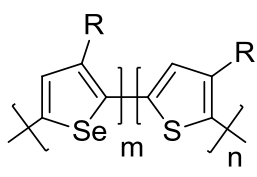

P3AS-b-P3AT

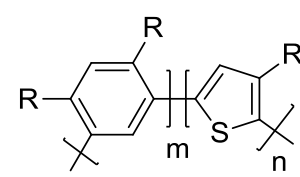

PMP-b-P3AT

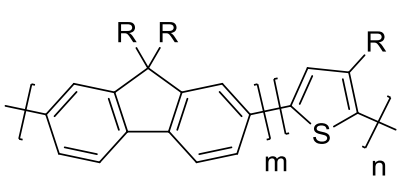

PF-b-P3AT

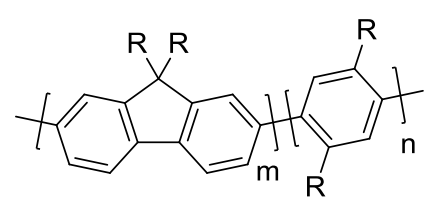

PF-b-PPP

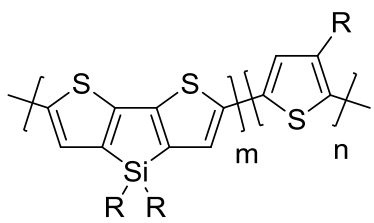

PDTS-b-P3AT

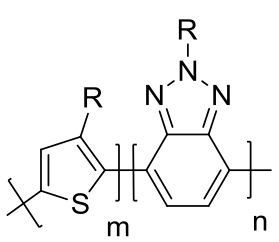

P3AT-b-PBT

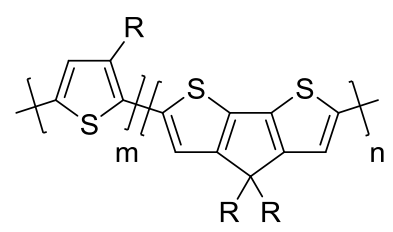

P3AT-b-PCPDT

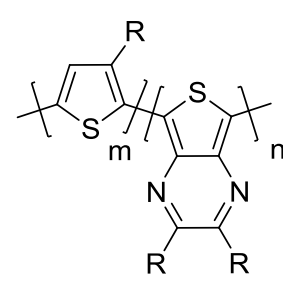

P3AT-b-PTP

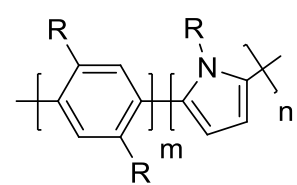

PPP-b-PP

$\mathrm{R}=$ alkyl, alkoxy side chain

Scheme 10: All-conjugated block copolymers with different aromatic moieties.

\subsubsection{Conjugated alternating copolymers}

CPs with a low bandgap are materials of great interest for opto-electronic applications. The synthesis of donor-acceptor $\pi$-conjugated alternating copolymers is a successful strategy to 
obtain this property and enables tailoring of the bandgap. ${ }^{194-196}$ The most popular approach towards the synthesis of alternating CPs uses a step-growth polycondensation mechanism, resulting in poor control over the molar mass and high dispersities. Examples of alternating CPs produced via controlled chain-growth polymerizations are limited. This can be attributed to the fact that the control of CTP relies on the complexation of the catalytic species to the $\pi$ conjugated system, which is significantly different for electron poor and electron rich monomers. A promising method towards alternating CPs is the synthesis of a monomer containing both building units. Kiriy and coworkers prepared alternating poly(fluorene-altbenzothiadiazole) $\left(\mathrm{P}(\mathrm{F}-\right.$ alt-BTDZ) $)$ via a SCTP of AB-monomers (Scheme 11). ${ }^{68}$ Although this approach is based on a chain-growth polymerization, control over the molar mass was not obtained. The research group of Bielawski succeeded in the synthesis of poly(thiophene-alt-pphenylene $) \quad(\mathrm{P}(3 \mathrm{HT}-\text { alt-PP }))^{197}$ and poly(5,6-difluorobenzotriazole-alt-3-hexylthiophene $)$ $(\mathrm{P}(\mathrm{FBTz}-\text { alt-3AT }))^{198}$ via KCTP. KCTP has further been applied for the synthesis of alternating CPs of P3HT and PTZ (P(3HT-alt-TZ) $)^{199}$, poly(3-alkylfuran) and P3AT (P(3AFalt-3AT) $)^{200}$ and P3AT and P3ASe (P(3AT-alt-3ASe $\left.)\right)^{201}$. The use of biaryl monomers for the production of alternating CPs was further extended to a transition-metal-free controlled polymerization. This approach has been introduced by Sanji et al. for the synthesis of P(PPalt $-\mathrm{T})^{148}$ and $\mathrm{P}(\mathrm{PP}-$ alt $-\mathrm{PE}){ }^{149}$ 


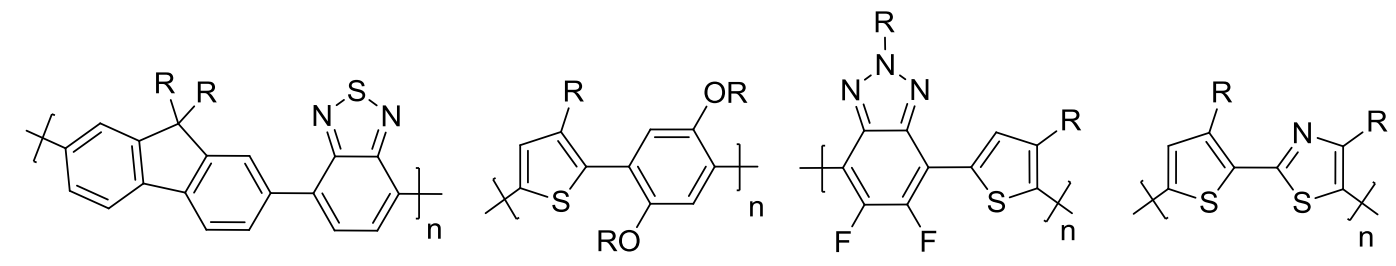

$\mathrm{P}(\mathrm{F}$-alt-BTDZ)

P(3AT-alt-PP)

P(FBTz-alt-3AT)

$\mathrm{P}(3 \mathrm{AT}-\mathrm{alt}-\mathrm{TZ})$<smiles>[R]c1cc(C(C)(C)C)oc1-c1cc([R])c(C([2H])([2H])[2H])s1</smiles>

P(3AF-alt-3AT)

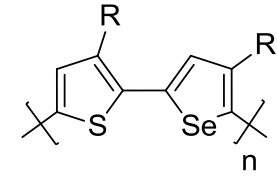

P(3AT-alt-3ASe)

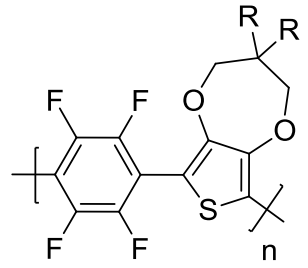

P(PP-alt-T)

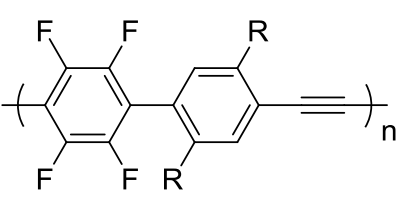

P(PP-alt-PE)

$\mathrm{R}=$ alkyl side chain

Scheme 11: Alternating conjugated copolymers prepared via chain-growth polymerization.

\subsubsection{Random/gradient copolymers}

Because the association of the catalyst to the polymer chain is the key to success in CTP, combining monomers with different electronic properties in a random conjugated polymer is difficult. The catalyst can stay associated to more electron rich sequences in the polymer and growth is discontinued. For example, when a mixture of thiophene and fluorene monomers is combined with a $\mathrm{Ni}(\mathrm{dppp}) \mathrm{Cl}_{2}$ catalyst, almost pure poly(thiophene) is formed and only marginal quantities of fluorene are incorporated. ${ }^{202}$ Hence, most examples of random copolymers synthesized using CTP are copolymers of thiophenes with different side chains. ${ }^{43,203-206}$ Thiophene was also copolymerized with selenophene and due to their similar electronic properties this resulted in a random copolymer as well. ${ }^{190,207}$ Palermo et al. were also able to synthesize a gradient copolymer of these two monomers via syringe pump addition of the selenophene monomer during the copolymerization. ${ }^{190}$ The same procedure was used to obtain gradient copolymers of two thiophenes with hexyl and hexyloxymethyl or hexyl and hexylbromide side chains. ${ }^{204,205}$ To be able to combine more electronically distinct 
monomers, Ni(II) diimine catalysts were developed by the research group of Seferos. Using this type of catalyst, even dithienosilole-benzotriazole random copolymers could be synthesized. ${ }^{208}$ Another possibility is to combine monomers with different electronic properties in biaryl or larger monomers and subsequently polymerize these in combination with other monomers. Using this method, a series of 'random' thiophene-phenylene copolymers was obtained. ${ }^{199,209}$ Finally, stepping away from CTP and using a controlled polymerization based on deactivation of the monomer is possible. The latter is much less dependent on the electronic properties of the monomer and e.g. the $\operatorname{Pd}(\operatorname{RuPhos})$ protocol was used to synthesize thiophene-fluorene gradient copolymers. ${ }^{202}$

\subsection{Termination and endcapping in CTP}

\subsubsection{Termination in KCTP}

When $\mathrm{Ni}(\mathrm{dppp}) \mathrm{Cl}_{2}$ or an external initiator is used to initiate the polymerization, the polymer chains will bear respectively a bromine atom or the functional group of the initiator (In) at the $\alpha$-end of the polymer chain. If termination is induced by the addition of $\mathrm{HCl}, \mathrm{H}$-terminated polymers (Scheme 12) are produced. In this way, control over the polymerization and perfect termination yields $\mathrm{Br} / \mathrm{H}$ - or $\mathrm{In} / \mathrm{H}$-terminated polymer chains. However, when dissociation of the catalyst during the polymerization occurs, the polymers will be $\mathrm{Br} / \mathrm{Br}-$ or $\mathrm{In} / \mathrm{Br}$ terminated. The dissociated catalyst can also initiate a new polymerization. After termination with $\mathrm{HCl}$, this transfer reaction leads to $\mathrm{H} / \mathrm{H}$-terminated polymer chains. 

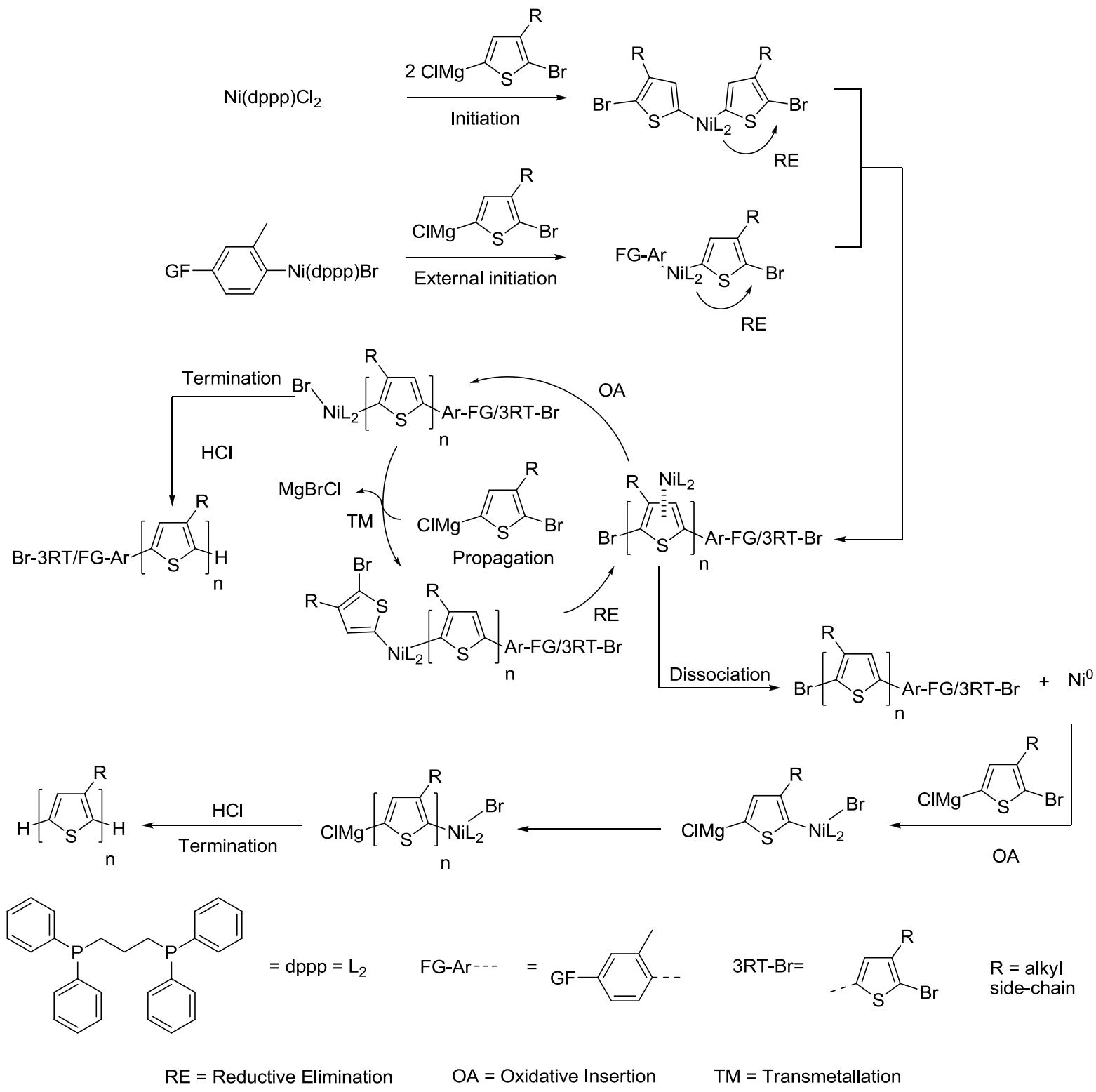

Scheme 12: The KCTP mechanism for the $\mathrm{Ni}(\mathrm{dppp}) \mathrm{Cl}_{2}$ and externally initiated polymerization of a substituted thiophene monomer. The initiator group (In) is replaced by FG-Ar.

Termination with $\mathrm{H}_{2} \mathrm{O}$ or $\mathrm{MeOH}$ leads to the same functionality at both chain ends and polymers with a molar mass double as expected. ${ }^{210,211}$ This indicates the occurrence of disproportionation reactions and yields $\mathrm{Br} / \mathrm{Br}$-terminated chains for $\mathrm{Ni}(\mathrm{dppp}) \mathrm{Cl}_{2}$-initiated polymerizations and In/In-terminated chains when an external Ni-initiator is used (Scheme 13). 


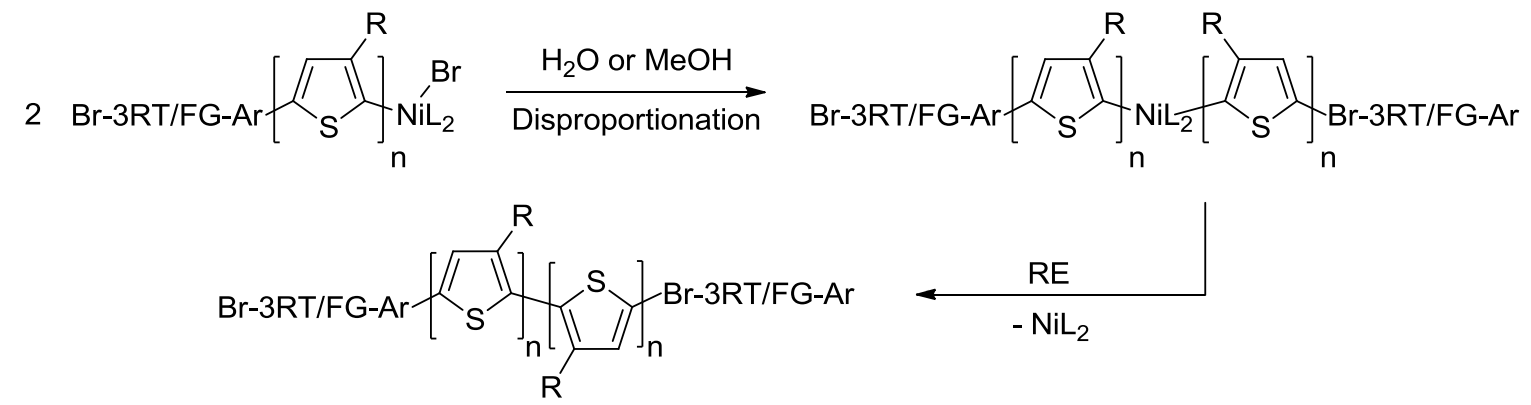

Scheme 13: Disproportionation in KCTP of 3-alkylthiophene monomers.

An incomplete Grignard metathesis reaction or an excess of Grignard reagent can result in a variety of side reactions at the chain ends, depending on the nature of the Grignard reagent used. ${ }^{211}$ For example, an excess of ${ }^{\mathrm{t}} \mathrm{BuMgCl}$ can react with the $\alpha-\mathrm{C}-\mathrm{Br}$ bond, which, after termination with $\mathrm{HCl}$, results in $\mathrm{H} / \mathrm{H}$-terminated chains. This side reaction could also occur when less bulky ${ }^{\mathrm{i}} \mathrm{PrMgCl}$ is used, but in this case also an ${ }^{\mathrm{i}} \mathrm{Pr}$-group could be built in, potentially leading to $\mathrm{H} / \mathrm{H}-, \quad \mathrm{In} /{ }^{i} \mathrm{Pr}-, \mathrm{H} /{ }^{\mathrm{i}} \mathrm{Pr}-$, and $\mathrm{Br} /{ }^{i} \mathrm{Pr}-$ terminated polymer chains. Determination of the end groups is generally done using ${ }^{1} \mathrm{H}$ NMR and MALDI-ToF analysis.

\subsubsection{Endcapping in KCTP}

Apart from the use of external initiators, also endcapping is an interesting strategy towards end-functionalized polymers. Endcapping in KCTP usually utilizes functionalized Grignard reagents, which are added to the reaction mixture at the end of the polymerization. After the oxidative addition of the Ni-catalyst into the terminal $\mathrm{C}-\mathrm{Br}$ bond, transmetalation with the endcapper occurs and the following reductive elimination yields the endcapped polymer.

The first endcapping experiments for KCTP were conducted by the group of McCullough. The $\mathrm{Ni}(\mathrm{dppp}) \mathrm{Cl}_{2}$ initiated polymerization of 3-alkylthiophenes was quenched with an excess of various Grignard reagents. ${ }^{212,213}$ They observed primarily mono-capped polymers with vinyl-, allyl-, ethynyl- and 3-aminophenylmagnesium bromide, but mainly di-capped chains with the other Grignard reagents (Scheme 14). For these endcappers, the catalyst reinserts in the $\alpha-\mathrm{C}-\mathrm{Br}$ bond after endcapping at the $\omega$-end, allowing a second endcapping. It can be 
hypothesized that the unsaturated Grignard reagents form a stable complex with the Nicatalyst. This prevents the reinsertion at the $\alpha-\mathrm{C}-\mathrm{Br}$ bond and hence a second endcapping. This hypothesis is supported by the observation that the addition of unsaturated molecules, such as styrene or 1-pentene, increases the ratio of mono- over di-capped polymer chains. ${ }^{214}$ Later, the group of Ueda observed only mono-capped chains when the polymerization was quenched with 3-[bis(trimethylsilyl)amino]phenylmagnesium bromide. Comparable effects can play a role since complexation of $\mathrm{Ni}$ (II) with bis(trimethylsilyl)amide ligands is known. ${ }^{215}$ Several other endcappers have been used to endcap $\mathrm{Ni}(\mathrm{dppp}) \mathrm{Cl}_{2}$ initiated 3-alkylthiophene polymerizations (Scheme 14). ${ }^{155,212-214,216-224}$ All these experiments yield di-capped polymer chains.

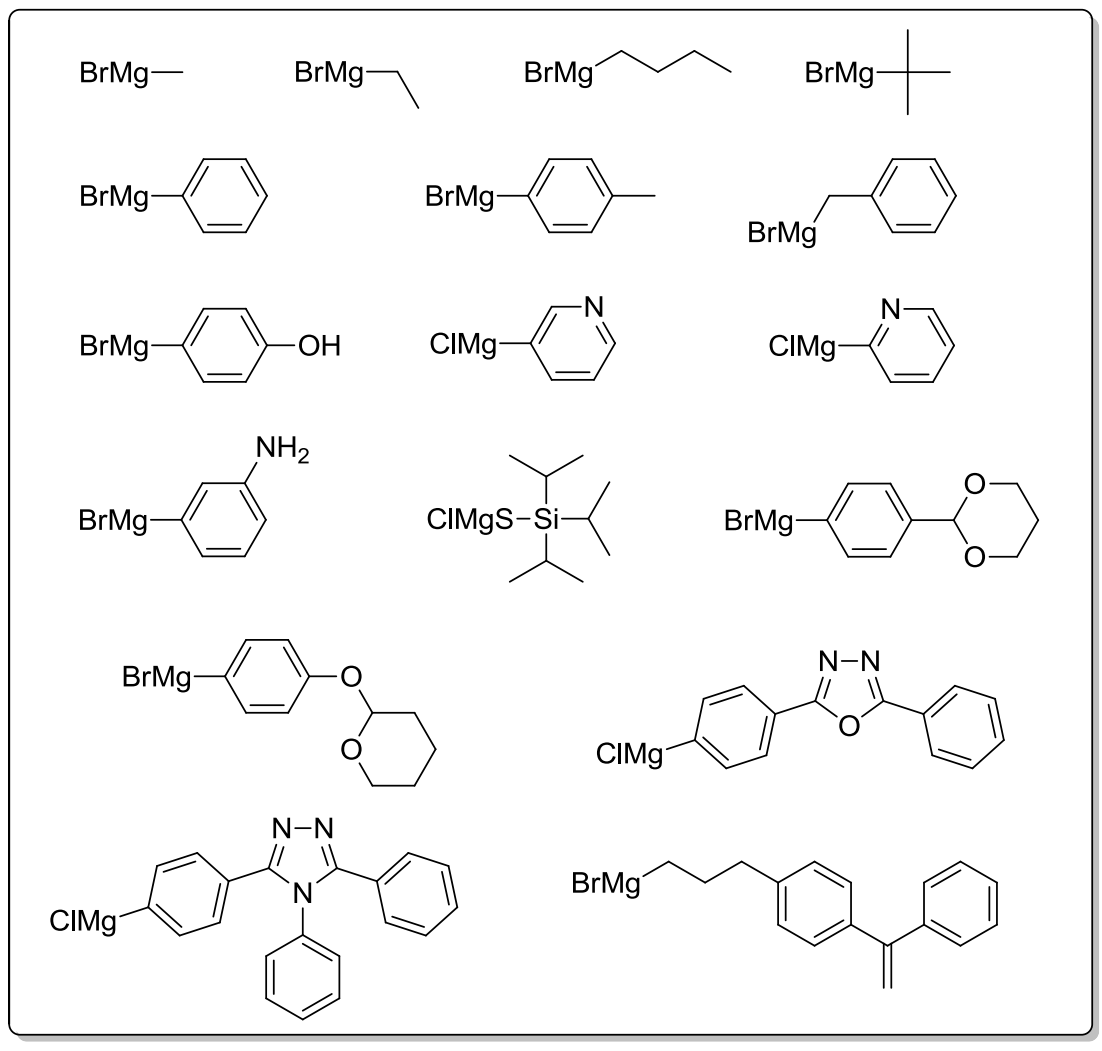

Dicapped

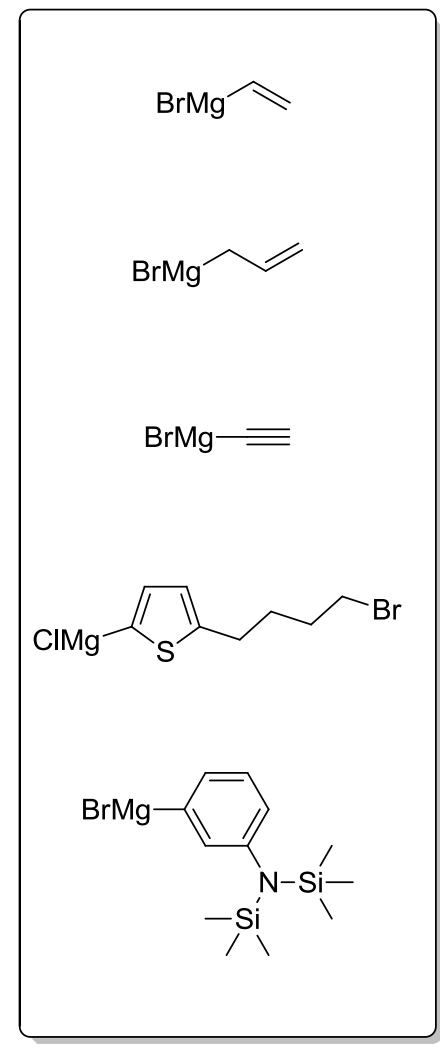

Monocapped

Scheme 14: Endcappers used in $\mathrm{Ni}(\mathrm{dppp}) \mathrm{Cl}_{2}$ initiated 3-alkylthiophene polymerizations. 
Next to the standard $\mathrm{Ni}(\mathrm{dppp}) \mathrm{Cl}_{2}$-catalyst, also external initiators have been used in similar endcapping research. ${ }^{225,226}$ The use of external initiators eliminates the possibility of reinsertion of the catalyst at the $\alpha$-chain end, yielding only mono-capped polymer. Unfortunately, often a significant amount of uncapped chains is present.

Interestingly, the group of Luscombe synthesized sulphur-capped poly(3-hexylthiophene), not by using a Grignard reagent, but through reaction with powdered sulphur. ${ }^{223}$ Both the $\mathrm{Ni}(\mathrm{dppp}) \mathrm{Cl}_{2}$ and externally initiated polymerizations yielded mono-capped chains, which can be attributed to the interaction of $\mathrm{S}^{-}$with the $\mathrm{Ni}$-catalyst.

Besides 3-alkylthiophene, also fluorene-based monomers have been used in endcapping experiments based on KCTP. ${ }^{118}$ However, endcapping of PF was proved more difficult than for P3ATs. This might be due to the lower degree of control over the polymerization of fluorene-based monomers.

\subsubsection{Termination and Endcapping in SCTP}

To obtain a controlled SCTP, an external Pd-initiator is necessary, conveniently capping the polymer with a functionalized aryl group (Ar) at the $\alpha$-chain end. After quenching with $\mathrm{HCl}$, $\mathrm{Ar} / \mathrm{H}-$ or $\mathrm{Ar} / \mathrm{Br}$-terminated chains are obtained (Scheme 15) ${ }^{8,62}$ When instead of $\mathrm{HCl}$, a boronic acid or ester is added to the polymerization mixture, a second functional group is installed at the $\omega$-chain end. ${ }^{63,66}$

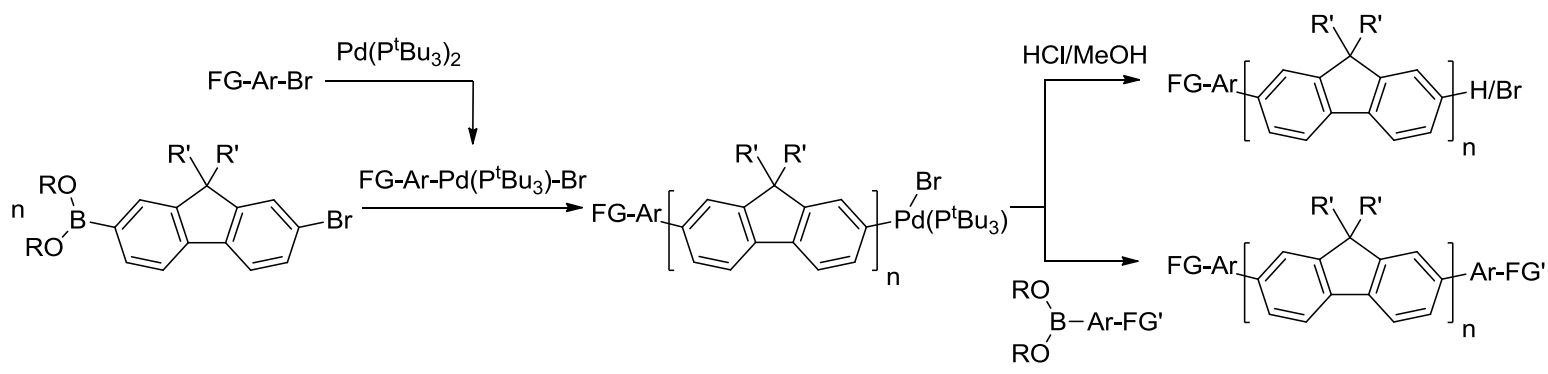

Scheme 15: General SCTP-mechanism for the synthesis of functionalized PFs. 


\section{Monomer deactivation}

Instead of relying on catalyst association, a controlled polymerization can also be achieved via deactivation of the monomer. This method has been applied to the synthesis of conjugated polymers by our group, more specifically using Negishi coupling reactions with a $\mathrm{Pd}(\mathrm{RuPhos})$ catalyst (Scheme 16). ${ }^{16}$ The bromozinc functionality on the monomer deactivates the $\mathrm{C}-\mathrm{Br}$ bond. As a consequence, the oxidative addition of the catalyst into this bond is prevented and no reaction occurs between monomer and catalyst. Hence, an initiator is required. This initiator is synthesized by combining the catalyst with a monomer lacking the deactivating bromozinc group. The $\mathrm{C}-\mathrm{Br}$ bond is then active and oxidative addition can occur. When this initiator is added to a batch of monomer, the polymerization will start with a transmetalation and subsequent reductive elimination. At this point, the catalyst can diffuse away from the polymer chain, so it is essential that it is stable in solution and very efficient in the next oxidative addition in a "dormant" polymer chain. Because of the deactivation of the monomer, no side reactions with other monomer occur. Once a new monomer is incorporated in the polymer chain, the deactivating functionality is no longer present. As a consequence, the $\mathrm{C}-\mathrm{Br}$ bond at the end of the polymer chain is always available for oxidative addition of the catalyst. In other words, the catalytic cycle can only continue at the end of a polymer chain, resulting in a chain-growth polymerization instead of a step-growth polymerization. 
Synthesis of the initiator
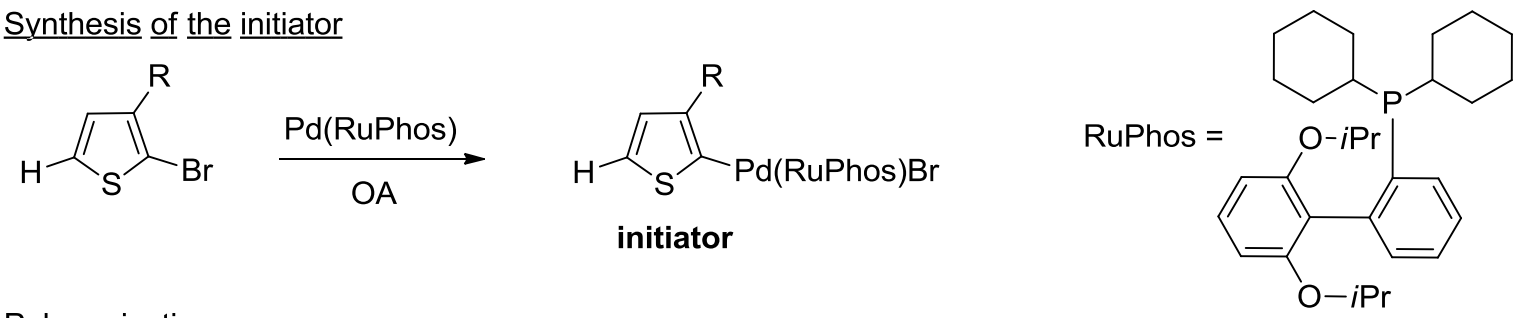

Polymerization
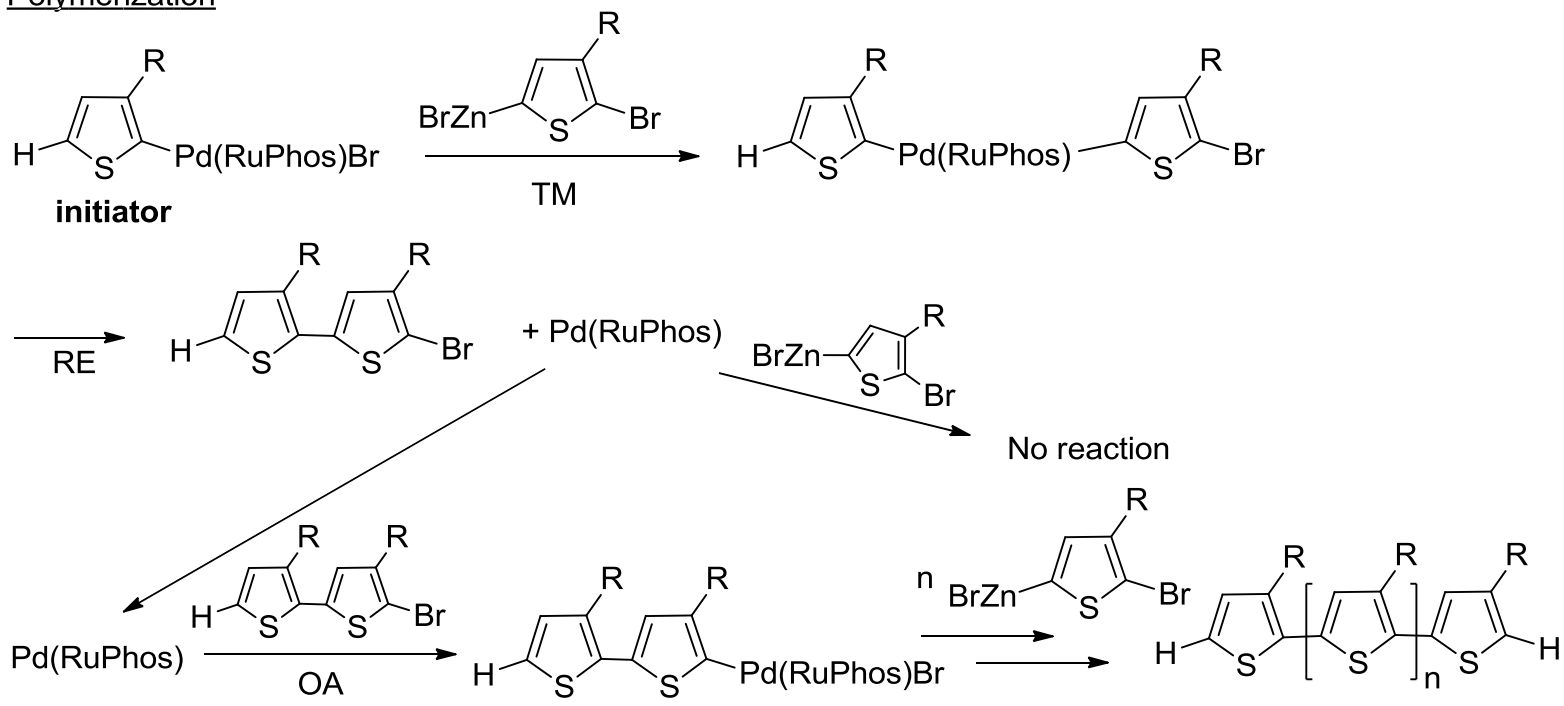

Scheme 16: Mechanism of the $\operatorname{Pd}(\mathrm{RuPhos})$ polymerization of 3-alkylthiophene.

To confer control to this chain-growth polymerization, termination and transfer reactions need to be retarded. Although termination reactions do not occur significantly, transfer reactions can pose a problem if no precautions are taken to work in very dry conditions. If moisture is present, the monomer is protonated and the deactivating functionality is lost. As a result, the $\mathrm{Pd}(\mathrm{RuPhos})$ catalyst can also insert in the $\mathrm{C}-\mathrm{Br}$ bond of this protonated monomer which acts as a transfer agent. However, if appropriately carried out, a controlled polymerization can be achieved.

Because association of the catalyst to the polymer chain is of no importance, the polymerization is much less dependent on the nature of the monomers. This advantage was exploited for the synthesis of multiblock copolymers with varying order of addition (Scheme 17). ${ }^{193}$ Using CTP, only one specific monomer sequence - with the monomers in order of increasing association with the catalyst, i.e. electron density - would be possible. Also, it 
enables the synthesis of random/gradient copolymers. While almost exclusively thiophene is incorporated in the polymer chain when thiophene and fluorene are copolymerized using $\mathrm{CTP}$, the $\mathrm{Pd}(\mathrm{RuPhos})$ protocol allows the formation of gradient copolymers. ${ }^{202}$
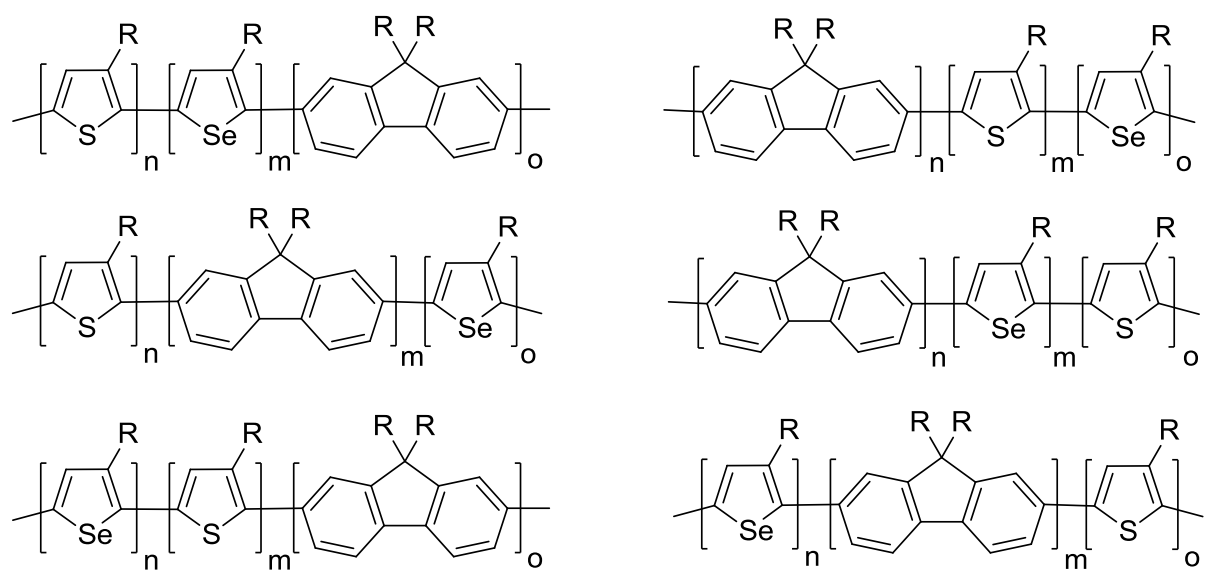

Scheme 17: Block copolymers synthesized through a varying order of addition using the $\mathrm{Pd}(\operatorname{RuPhos})$ protocol.

Furthermore, the larger size of the Pd atom with respect to Ni eases the formation of head-tohead couplings. Consequently, using both isomers of the thiophene monomer (2-bromo-5bromozinc-3-alkylthiophene and 5-bromo-2-bromozinc-3-alkylthiophene), P3AT with a controlled degree of regio-irregularity could be synthesized (Scheme 18). ${ }^{203,227}$ While this is in principle also possible with CTP, the most used catalysts, $\mathrm{Ni}(\mathrm{dppp}) \mathrm{X}_{2}$ and $\mathrm{Ni}(\mathrm{dppe}) \mathrm{X}_{2}$, always provide head-to-tail P3AT.<smiles>[R]c1cc(Br)sc1[Br+]Br</smiles>

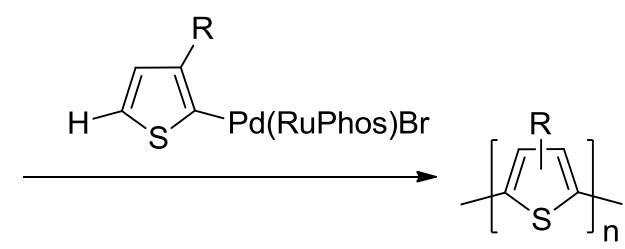

Scheme 18: Synthesis of poly(thiophene)s with a controlled regioregularity. 


\section{Advanced Topologies}

The focus in the field of conjugated polymers has mostly been on the development of linear structures. However, in recent years efforts were made to synthesize structures with different and more advanced topologies. Different architectures, e.g. graft copolymers, cyclic, branched and star polymers, have been explored.

\subsection{Graft copolymers}

A graft copolymer is a comb-shaped polymer consisting of a polymer backbone with several polymer side chains along its length. Graft copolymers can show interesting properties in terms of supramolecular assembly and might be ideal materials for the preparation of organic electronics. ${ }^{228-233}$

There are three methods to prepare graft copolymers: grafting from, grafting through and grafting onto, as shown in Scheme 19. ${ }^{234}$ All three methods have been used to prepare allconjugated graft copolymers.

a)
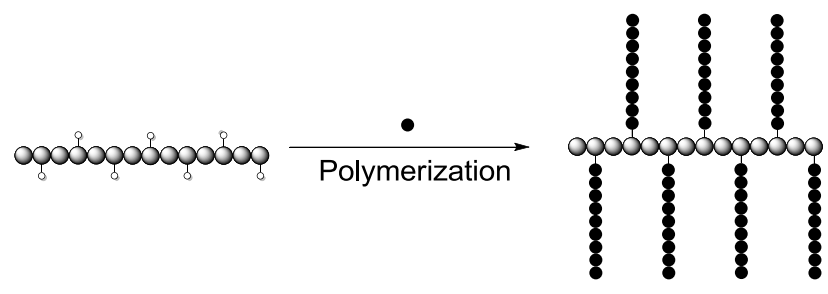

b)
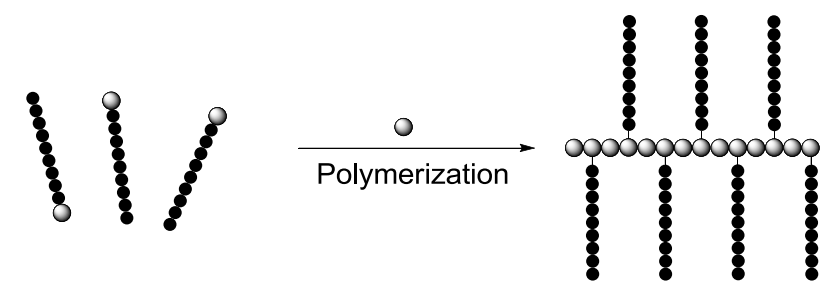

c)
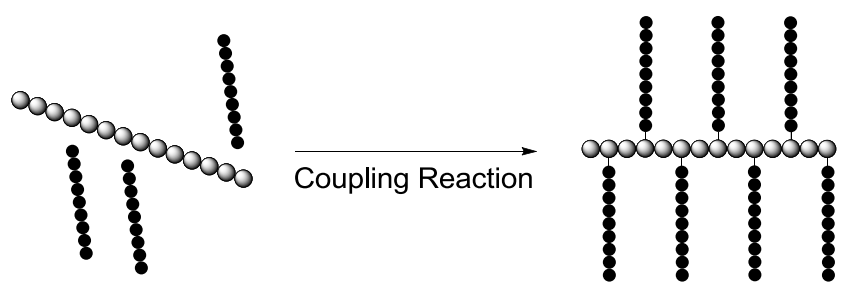

Scheme 19: a) grafting from; b) grafting through; c) grafting onto. 
In the grafting from method, initiating units are created along the polymer backbone. A second polymerization can then be initiated from the polymer backbone, creating a graft copolymer. The number of side chains can be predicted if every initiating unit actually initiates a new polymer chain. A major downside of the grafting from method is the difficult characterization of the polymer side chains.

The first all-conjugated graft copolymer was made by Wang et al. using the grafting from method. ${ }^{230}$ Two different graft copolymers were made, both with thiophene side chains, but one with a thiophene-based backbone (32) and one with a naphthalene diimide thiophene backbone (33) (Scheme 20). After the polymerization of the backbone, the initiating groups were formed by the reaction with $\mathrm{Ni}(\mathrm{COD})_{2}$ and $\mathrm{PPh}_{3}$. After the ligand exchange with dppp, the KCTP was started, resulting in an all-conjugated graft copolymer with side chains synthesized in a controlled fashion. After the grafting reaction a clear increase in molar mass and decrease in dispersity was seen. This decrease can be attributed to the controlled character of the KCTP mechanism.

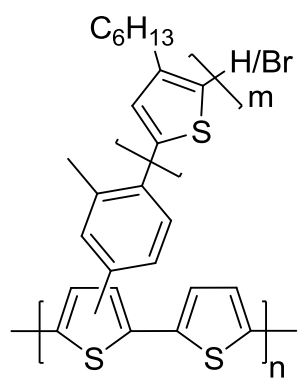

32

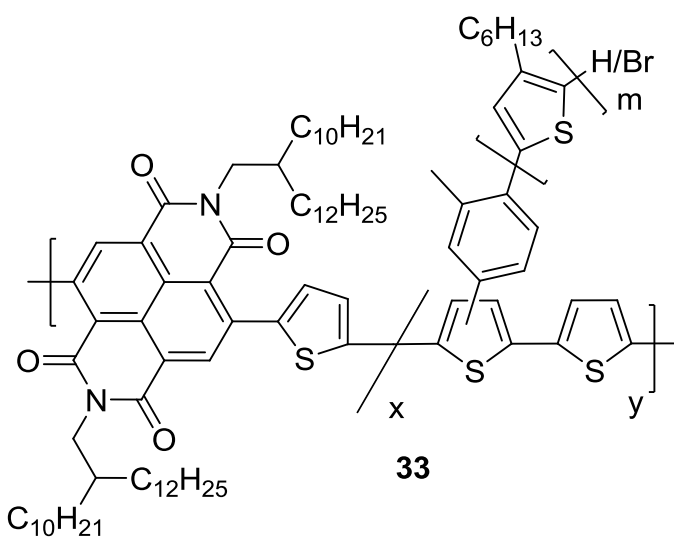

Scheme 20: Graft copolymers prepared by the grafting from technique.

In the grafting through method, the side chain polymers are synthesized first and are commonly referred to as macromonomers. These macromonomers are then polymerized, resulting in a graft copolymer. The group of Luscombe was the first to synthesize a 
conjugated graft copolymer using this grafting through approach (Scheme 21). ${ }^{231}$ The polymer side chain in this graft copolymer was again poly(3-hexylthiophene), synthesized in a controlled fashion using the KCTP mechanism with an external initiator. This initiator was based on 9-(4-chloro-3-methylphenyl)-2,7-bis-(tetramethyl-1,3,2-dioxaborolan-2-yl)-9Hcarbazole and subsequent treatment with $\mathrm{Ni}(\mathrm{COD})_{2}, \mathrm{PPh}_{3}$ and dppp resulted in the initiator. Addition of 2-chloromagnesio-5-bromo-3-hexylthiophene to the initiator led to a controlled polymerization. The incorporation of the initiator was confirmed by MALDI-ToF analysis. After the macromonomers were obtained, they were copolymerized in a non-controlled fashion with a diketopyrrolopyrrole derivative compound using Suzuki-Miyaura couplings (34).

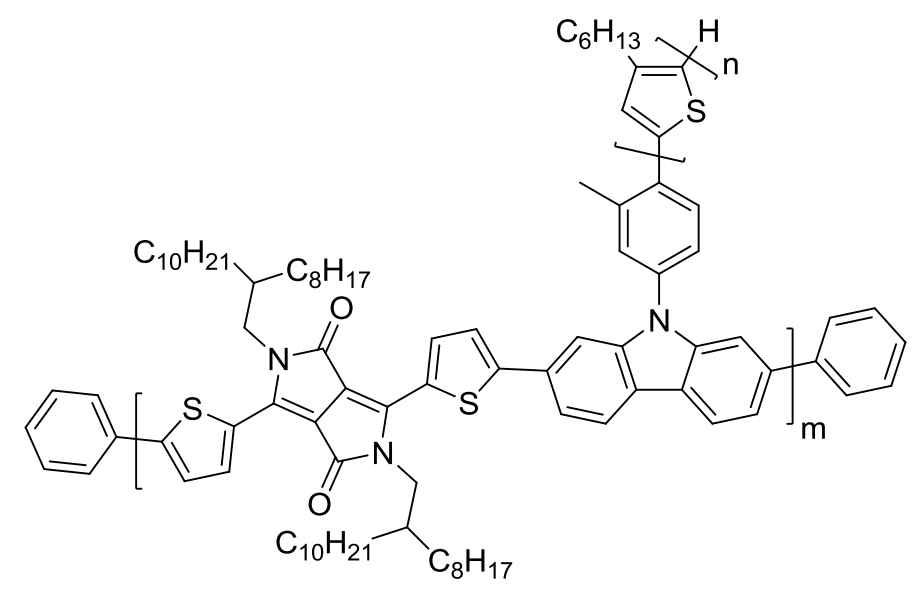

34

Scheme 21: Graft copolymers prepared by the grafting through technique.

In the grafting onto method, the polymer backbone and the polymer side chains are prepared separately and coupled afterwards. Both polymers are equipped with functional groups to make this coupling possible. The side chains are equipped with just one functional group at the chain end, while the backbone has several functional groups spread across its entire length. Our group synthesized two different conjugated graft copolymers, both using the grafting onto method (Scheme 22). ${ }^{232,233}$ The first one consists of a PPE backbone, 
polymerized in a non-controlled fashion using Sonogashira-couplings (35). The polymer side chains are poly(3-hexylthiophene), synthesized in a controlled way via the KCTP mechanism. This polymerization was terminated with ethynylmagnesium bromide, resulting in an acetylene endcapped polymer. ${ }^{213}$ The backbone units are equipped with azide functionalities and in this way the graft copolymer can be prepared by a CuAAC click reaction. After the grafting reaction, the graft copolymer was separated from the non-reacted homopolymers by preparative GPC. Because of this purification, the resulting polymers all showed a low dispersity between 1.2 and 1.4. The grafting density of the graft copolymers was in accordance with the aimed values for the lower grafting densities (10 and 25\%). However, when higher grafting densities were aimed for, the graft copolymers differed more strongly (38\% instead of $50 \%$ and $50 \%$ instead of $100 \%$ ). This can be explained by increased sterical hindrance when introducing more and more polymer side chains.

Next, our group synthesized a second graft copolymer using the CuAAC click reaction (36). It consisted of a thiophene backbone with azide moieties on the alkyl side chain and was prepared by KCTP. PF was used as polymer side chains. It was prepared using a method described by Yokozawa et al. ${ }^{8,186,235,236} \mathrm{PhPd}\left(\mathrm{P}^{t} \mathrm{Bu}_{3}\right) \mathrm{Br}$ was used as initiator and $p$-boronic phenylmethanol as endcapper. This polymerization method normally results in a controlled polymerization with well-defined end groups. However, in this case a mixture of several end groups was obtained. The end-capped PF can be functionalized with an ethynyl function, making the CuAAC click reaction possible for the preparation of the graft copolymer. Also for this polymer, the grafting density was lower than anticipated. 

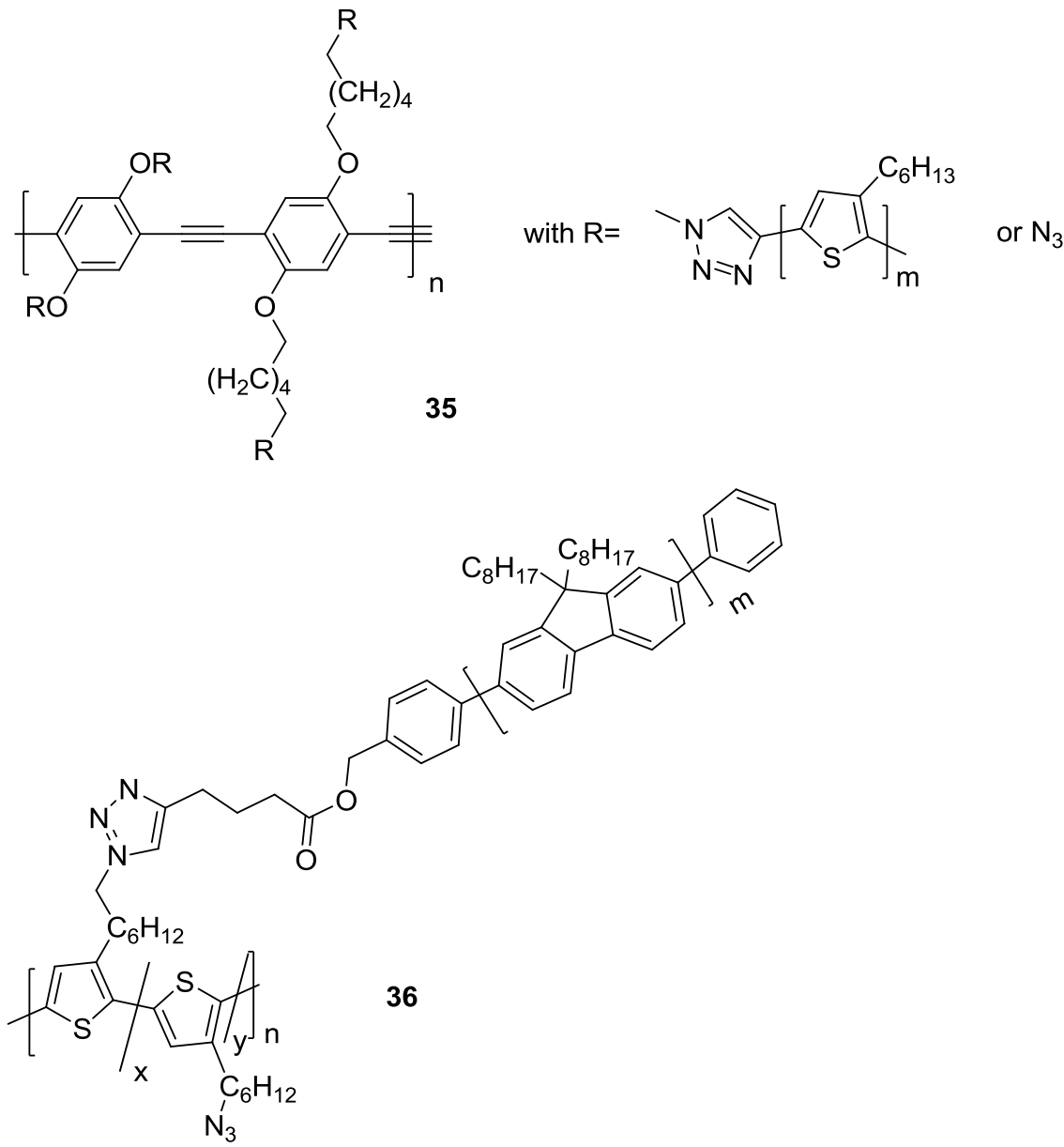

Scheme 22: Graft copolymers prepared by the grafting onto technique.

\subsection{Cyclic polymers}

To prepare a macrocyclic polymer, the polymer needs to be equipped with well-defined end groups, capable of reacting with each other. Several macrocyclic conjugated polymers have already been prepared in a non-controlled fashion. ${ }^{237-244}$ The first and only conjugated macrocycle synthesized in a controlled way, was made by Coulembier et al. ${ }^{245}$ The authors opted for poly(3-hexylthiophene) prepared by KCTP with a keto-functionalized external initiator. After polymerization, In/H-terminated polymers with a low dispersity were obtained, as demonstrated by MALDI-ToF and GPC analysis. This polymer was then endfunctionalized with a post-polymerization Vilsmeier-reaction to introduce an aldehyde 
function in very high yield, as previously reported by McCullough. ${ }^{246,247}$ The linear polymer was then cyclized using an aldol condensation under very diluted conditions (37).

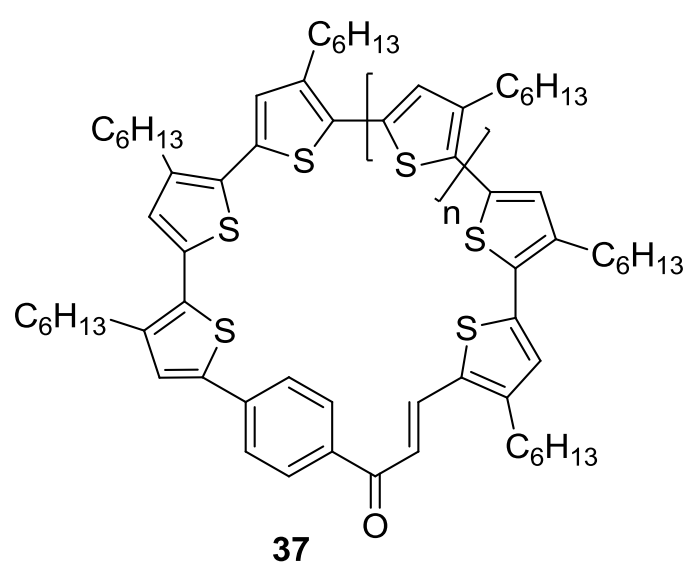

Scheme 23: Macrocyclic conjugated polymer synthesized in a controlled way.

\subsection{Branched polymers}

A wide range of conjugated branched, hyperbranched and even dendritic structures have been described in literature. ${ }^{248-251}$ Most of these structures were prepared using non-controlled polymerization methods. However, some efforts have been made to prepare branched and hyperbranched conjugated polymers in a controlled fashion.

$\mathrm{Xu}$ et al. were the first to synthesize a hyperbranched poly(thiophene) using a KCTP-like mechanism. ${ }^{252}$ 2,3-dibromothiophene was treated with $\mathrm{LDA}$ and $\mathrm{MgBr}_{2}$. The resulting Grignard reagent was then added to $\mathrm{Ni}(\mathrm{dppp}) \mathrm{Cl}_{2}$. Unfortunately, the resulting polymer was poorly soluble. This problem was, however, solved by the addition of hexylmagnesium bromide and an extra amount of $\mathrm{Ni}(\mathrm{dppp}) \mathrm{Cl}_{2}$ after the polymerization (38). Scheuble et al. used a similar approach to synthesize branched poly(thiophene)s using 5,5"-dibromo$2,2^{\prime}: 3^{\prime}, 2^{\prime \prime}$-terthiophene. ${ }^{253}$ The obtained polymer (39) was compared to branched poly(thiophene) prepared after Grignard metathesis reaction and polymerization of 5,5',5"tribromo-2,2':3',2"-terthiophene (40). The Grignard metathesis reaction led to different regioisomers which were incorporated in different amounts. A hyperbranched poly(thiophene) has 
also been synthesized by Okamoto et al. using the KCTP mechanism (41). ${ }^{254}$ The branched polymers are shown in Scheme 24.

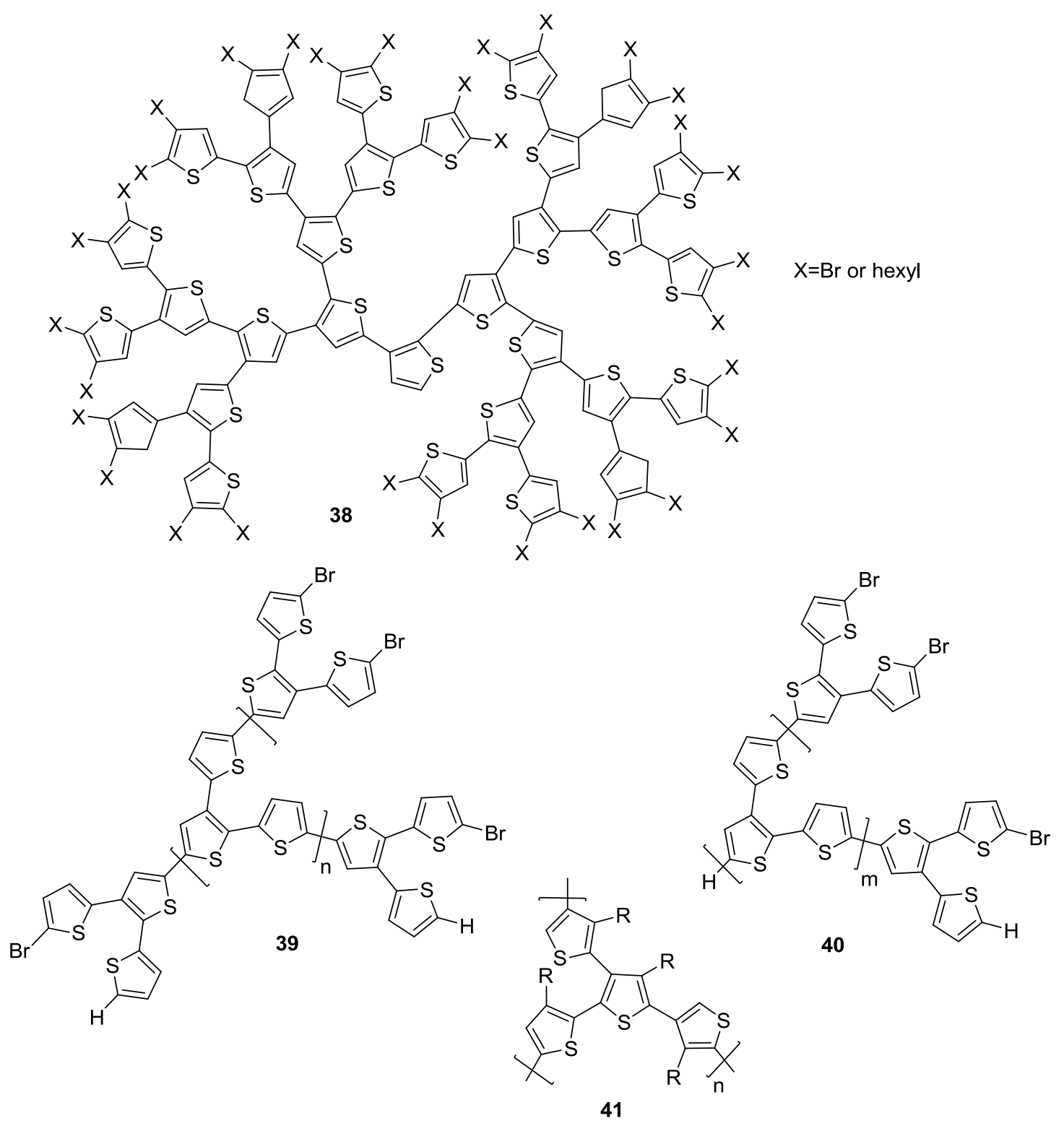

Scheme 24: Hyperbranched poly(thiophene)s synthesized with KCTP.

A third method to synthesize branched poly(thiophene)s was developed by $\mathrm{Tu}$ et $a l .{ }^{255} \mathrm{~A}$ mixture of two thiophene monomers, after treatment with methylmagnesium bromide, was polymerized (42): 'linear' 2,5-dibromo-3-hexylthiophene and 'branched' 2,5,2',5'tetrabromo-3,3'-bithiophene. Polymerization with an increasing amount of branched 
monomer led to more soluble polymers compared to linear P3HT with similar molar masses (Scheme 25).

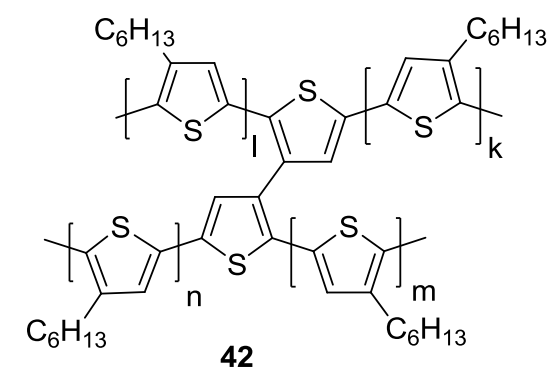

Scheme 25: Branched poly(thiophene) synthesized from a mixture of two different monomers.

The controlled synthesis of hyperbranched conjugated polymers was also done with SCTP (Scheme 26). A hyperbranched PPP (43) was synthesized in this way. ${ }^{258}$ There was, however, no complete control over the polymerization and properties of both chain and step-growth mechanisms were present.<smiles>CC(C)(C)c1cc(-c2cc(-c3cc(-c4cc(C(C)(C)C)cc(C(C)(C)C)c4)cc(-c4cc(C(C)(C)C)cc(C(C)(C)C)c4)c3)cc(-c3cc(C(C)(C)C)cc(C(C)(C)C)c3)c2)cc(-c2cc(-c3cc(-c4cc(C(C)(C)C)cc(C(C)(C)C)c4)cc(-c4cc(C(C)(C)C)cc(C(C)(C)C)c4)c3)cc(C(C)(C)C)c2)c1</smiles>

Scheme 26: Hyperbranched conjugated polymer synthesized with SCTP.

\subsection{Star-shaped polymers}

A special category of branched polymers are star polymers, which consist of linear polymer chains attached to a core. There are two general strategies to synthesize star polymers: an arm first approach or a core first approach. 
The arm first approach is based on a multifunctional coupling agent that serves as the core of the star polymer. Multiple polymer chains can then react with this core. Using this approach, a conjugated star polymer was prepared by Kim et al. ${ }^{260}$ Poly(3-hexylthiophene) was endcapped with an allyl moiety, which was transformed into a chlorophenylacetate initiator. The resulting macroinitiator was then used in the ATRP (Atom transfer radical polymerization) of divinylmonomers, creating a crosslinked polymer core with pending thiophene arms (44) as shown in Scheme 27.

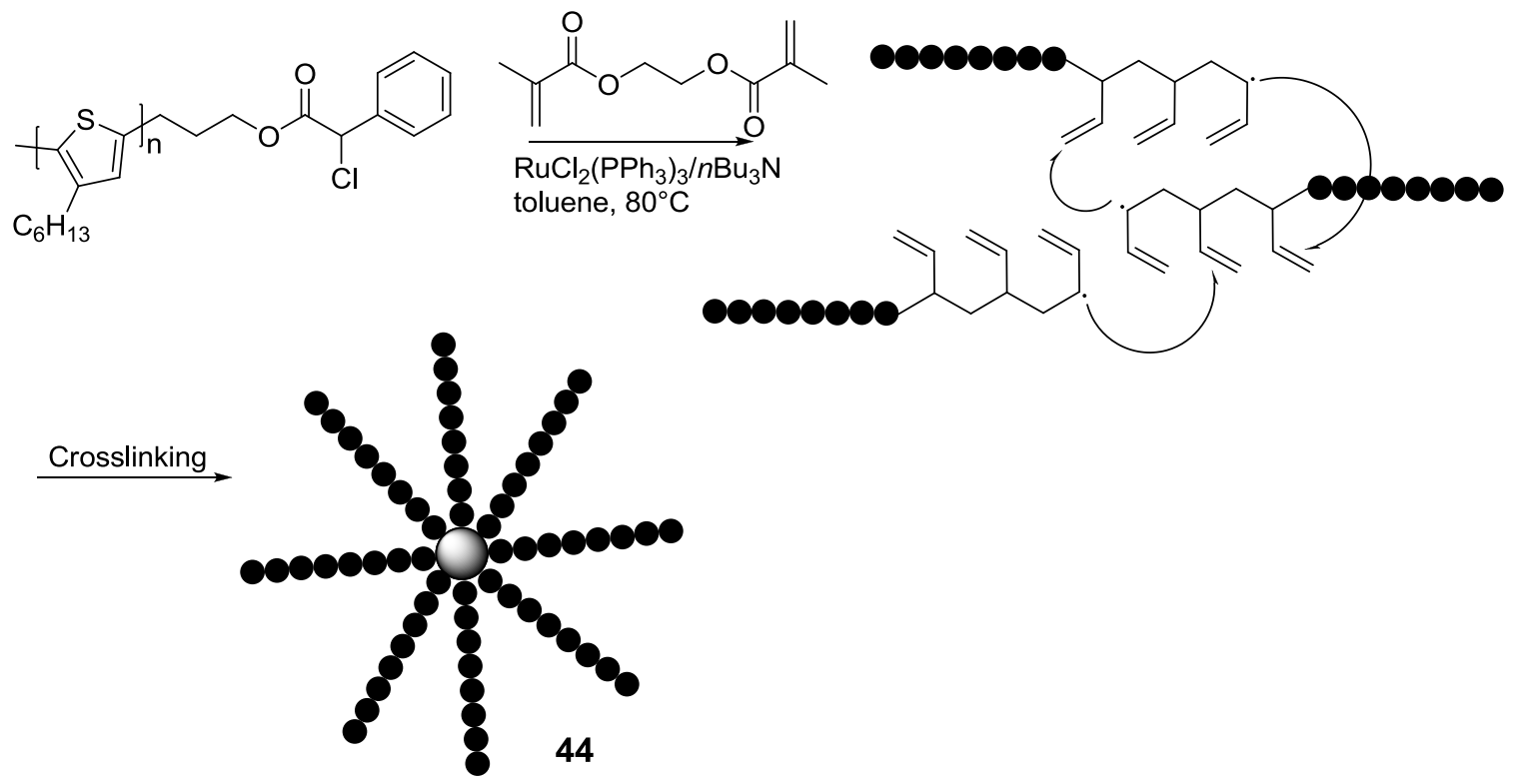

Scheme 27: Synthetic method to prepare a star polymer using the arm-first approach.

In the core first approach, the core is synthesized first and then the polymerization is initiated at several locations in the core periphery. This approach has been used a few times for the synthesis of conjugated star polymers. The first example was published in 1997 by Wang et $a l .{ }^{261}$ The star polymer (45) consisted of a hyperbranched 1,3,5-polyphenylene core equipped with terminal $\mathrm{C}-\mathrm{Br}$ bonds. These $\mathrm{C}-\mathrm{Br}$ bonds were reacted with 2-bromomagnesiothiophene, followed by NBS, to minimize the differences in reactivity between the core and the growing polymer chains. Approximately $52 \%$ of the terminal $\mathrm{C}-\mathrm{Br}$ bonds were substituted with a 
thiophene unit. The thiophene branches were then synthesized by the addition of $\mathrm{Ni}(\mathrm{dppp}) \mathrm{Cl}_{2}$ and 2-bromo-5-bromomagnesio-3-hexylthiophene (Scheme 28).

An alternating star copolymer was also made by Wang et al. using a hyperbranched poly(triphenylamine) core (46). The polymer arms were thiophene-phenylene alternating copolymers synthesized by KCTP of 3,4-ethylenedioxythiophene-didodecyloxybenzene monomers. $^{262}$

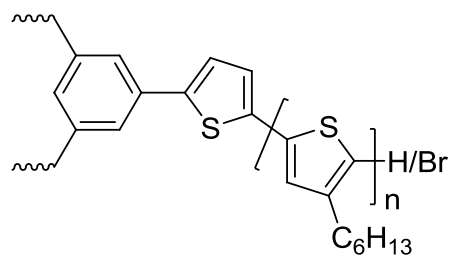

45

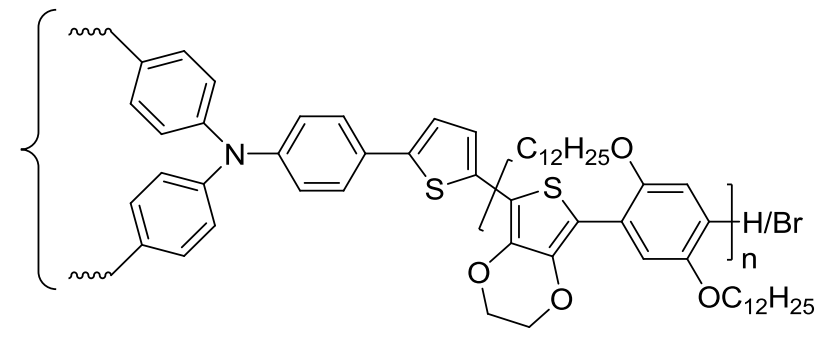

46

Scheme 28: Star polymers prepared by the core first approach.

The same approach was also used by Senkovskyy et al. to synthesize a thiophene star polymer. ${ }^{72}$ The authors used a hexa( $p$-bromophenyl)benzene-based core to which Ni(bipy)Et 2 was added to create a multifunctional initiator with six initiating units (47). Poly(3-hexyl)thiophene arms were then initiated from this core. Yuan et al. synthesized a similar poly(3-hexylthiophene) star polymer starting from two and three armed biphenyl cores using first $\mathrm{Ni}(\mathrm{COD})_{2}$ and $\mathrm{PPh}_{3}$, after which dppp was added to obtain the initiator $(\mathbf{4 8 ,}, \mathbf{4 9}) .^{70}$ All three multifunctional initiators are shown in Scheme 29. 


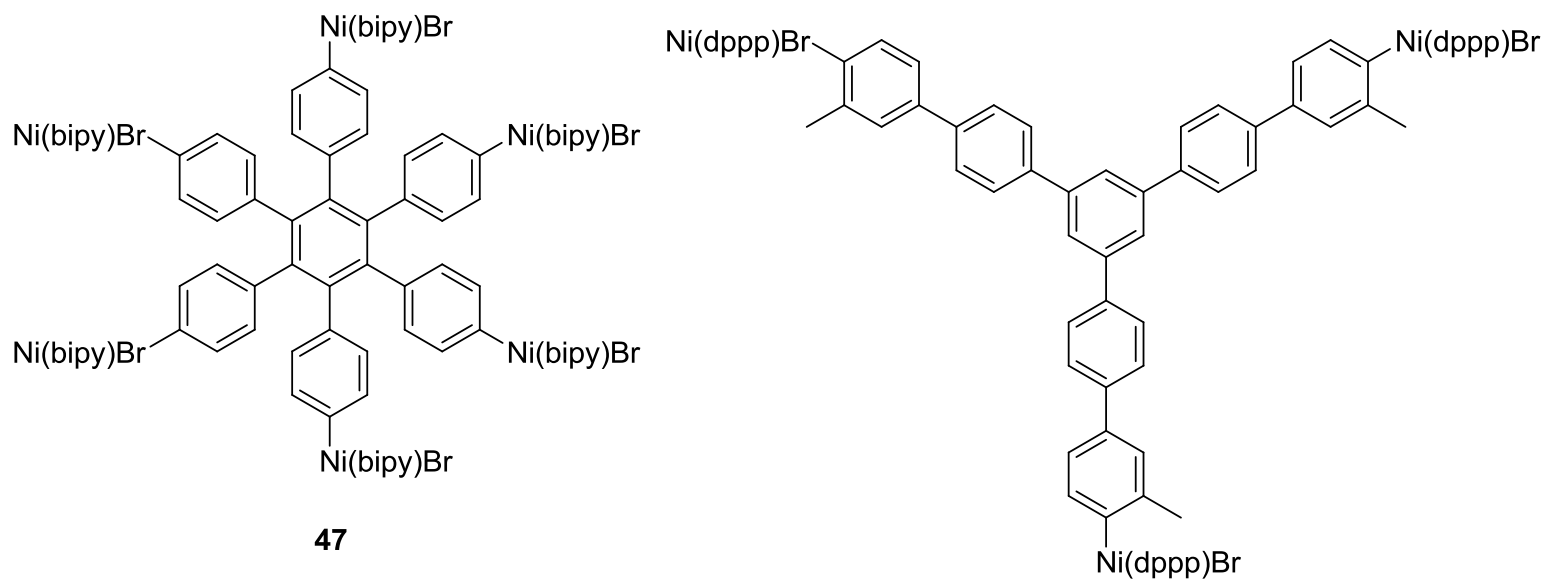

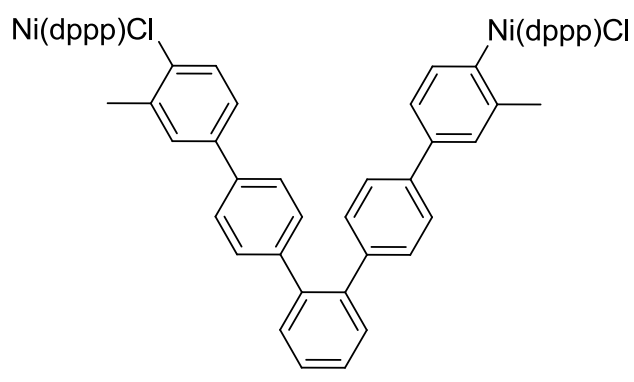

48

49

Scheme 29: Multifunctional initiators to prepare star polymers using the core first approach.

A PF star polymer (50) was recently prepared by Fischer et al. using the core first approach. ${ }^{71}$ A tetra(4-iodophenoxy)-substituted terrylene diimide was used as the core. The terminal iodine functions can be transformed in situ into a functional initiator using $\operatorname{Pd}(\mathrm{dba})_{2}$ and $\mathrm{P}^{\mathrm{t}} \mathrm{Bu}_{3}$. Addition of the 2-bromo-7-pinacol boronic ester fluorene monomer leads to a controlled synthesis of the PF star polymer (Scheme 30). 


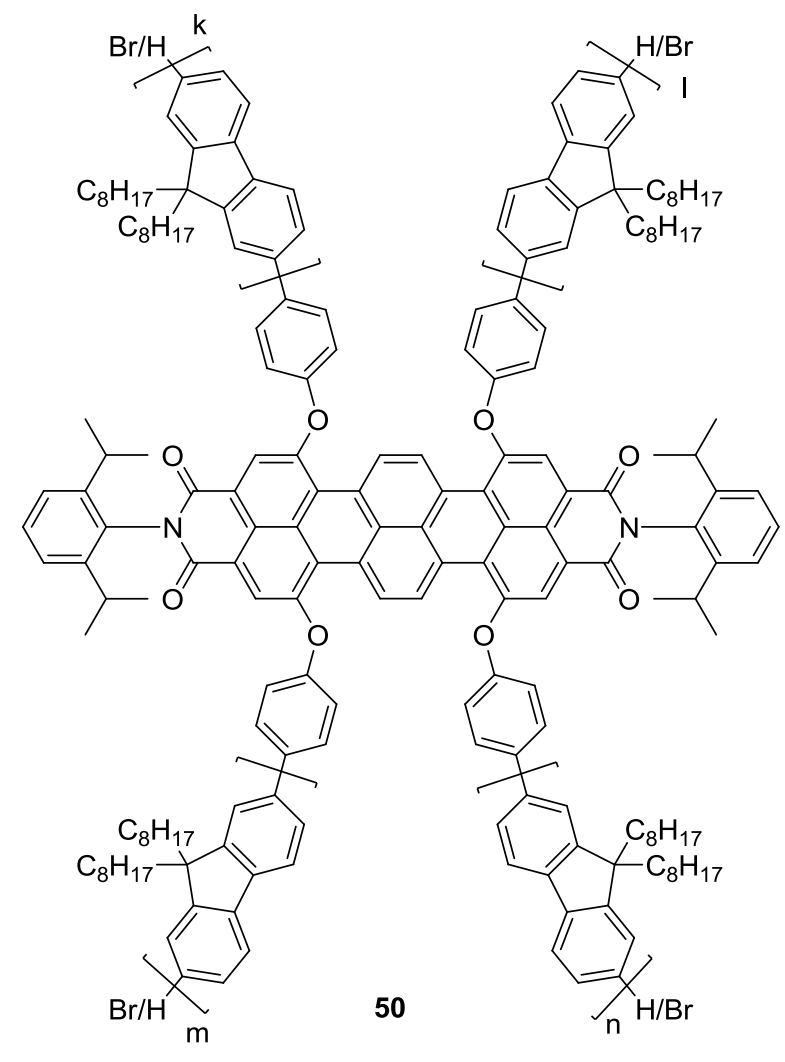

Scheme 30: PF star polymer synthesized a controlled synthesis.

\section{Conclusions and outlook}

Since P3HT was prepared using $\mathrm{Ni}(\mathrm{dppp}) \mathrm{Cl}_{2}$ in 1993, an enormous progress has been made. The discovery that such polymerization actually proceeds via a controlled chain-growth mechanism marked the beginning of this journey. Since then, many different monomers have been polymerized in a controlled way using different (organometallic) reactions. Copolymers - random, gradient and block - have been prepared and shown to have unique properties. Also CPs with new topologies have been realized.

However, challenges still remain. The number of monomers that can actually be polymerized in a controlled way remains limited: most of them are based on thiophene. The simple replacement of thiophene by phenylene already makes the polymerization a lot more difficult. Electron deficient or even more complex monomers are notoriously difficult to polymerize in a controlled way. Simple structures as copolymers are often still inaccessible. It can be stated 
that there is still a long way to go to reach a fully living polymerization of CPs with all related possibilities.

\section{Acknowledgements}

We are grateful to the Onderzoeksfonds K.U.Leuven/Research Fund K.U.Leuven and the Fund for Scientific Research (FWO-Vlaanderen) for financial support. L.V. and T.H. are a doctoral fellows of the Fund for Scientific Research (FWO-Vlaanderen) and W.C. is a doctoral fellow of the Institution for Innovation by Science and Technology (IWT).

\section{References}

(1) Grisorio, R.; Suranna, G. P. Polym. Chem. 2015, 6 (45), 7781-7795.

(2) Yokozawa, T.; Ohta, Y. Chem. Rev. 2016, 116 (4), 1950-1968.

(3) Yokoyama, A.; Miyakoshi, R.; Yokozawa, T. Macromolecules 2004, 37 (4), 1169-1171.

(4) Sheina, E. E.; Liu, J.; Iovu, M. C.; Laird, D. W.; McCullough, R. D.; Lovu, M. C.; Laird, D. W.; McCullough, R. D. Macromolecules 2004, 37 (10), 3526-3528.

(5) Bryan, Z. J.; McNeil, A. J. Chem. Sci. 2013, 4 (4), 1620.

(6) Senkovskyy, V.; Tkachov, R.; Komber, H.; John, A.; Sommer, J.-U.; Kiriy, A. Macromolecules 2012, 45 (19), 7770-7777.

(7) Willot, P.; Koeckelberghs, G. Macromolecules 2014, 47 (24), 8548-8555.

(8) Yokoyama, A.; Suzuki, H.; Kubota, Y.; Ohuchi, K.; Higashimura, H.; Yokozawa, T. J. Am. Chem. Soc. 2007, 129 (23), 7236-7237.

(9) Kang, S.; Ono, R. J.; Bielawski, C. W. J. Am. Chem. Soc. 2013, 135 (13), 4984-4987.

(10) Qiu, Y.; Mohin, J.; Tsai, C.-H.; Tristram-Nagle, S.; Gil, R. R.; Kowalewski, T.; Noonan, K. J. T. Macromol. Rapid Commun. 2015, 36 (9), 840-844.

(11) Fuji, K.; Tamba, S.; Shono, K.; Sugie, A.; Mori, A. J. Am. Chem. Soc. 2013, 135 (33), 1220812211.

(12) Nanashima, Y.; Yokoyama, A.; Yokozawa, T. Macromolecules 2012, 45 (5), 2609-2613.

(13) Groombridge, B. J.; Goldup, S. M.; Larrosa, I. Chem. Commun. 2015, 51 (18), 3832-3834.

(14) Bryan, Z. J.; Hall, A. O.; Zhao, C. T.; Chen, J.; McNeil, A. J. ACS Macro Lett. 2016, 5 (1), 6972.

(15) Yokoyama, A.; Yokozawa, T. Macromolecules 2007, 40 (12), 4093-4101.

(16) Verswyvel, M.; Verstappen, P.; De Cremer, L.; Verbiest, T.; Koeckelberghs, G. J. Polym. Sci. Part A Polym. Chem. 2011, 49 (24), 5339-5349.

(17) Yokozawa, T.; Yokoyama, A. Chem. Rev. 2009, 109 (11), 5595-5619.

(18) Iovu, M. C.; Sheina, E. E.; Gil, R. R.; McCullough, R. D. Macromolecules 2005, 38 (21), 8649-8656.

(19) Miyakoshi, R.; Yokoyama, A.; Yokozawa, T. J. Am. Chem. Soc. 2005, 127 (49), 17542-17547.

(20) Komber, H.; Senkovskyy, V.; Tkachov, R.; Johnson, K.; Kiriy, A.; Huck, W. T. S.; Sommer, M. Macromolecules 2011, 44 (23), 9164-9172.

(21) Tkachov, R.; Senkovskyy, V.; Komber, H.; Kiriy, A. Macromolecules 2011, 44 (7), 20062015.

(22) Yokozawa, T.; Ohta, Y. Chem. Commun. 2013, 49 (75), 8281-8310. 
(23) Lamps, J.-P.; Catala, J.-M. Macromolecules 2009, 42 (19), 7282-7284.

(24) Beryozkina, T.; Senkovskyy, V.; Kaul, E.; Kiriy, A. Macromolecules 2008, 41 (21), 7817 7823.

(25) Yokozawa, T.; Yokoyama, A. Chem. Rev. 2009, 109 (11), 5595-5619.

(26) Qiu, Y.; Worch, J. C.; Fortney, A.; Gayathri, C.; Gil, R. R.; Noonan, K. J. T. Macromolecules 2016, 49 (13), 4757-4762.

(27) Doubina, N.; Ho, A.; Jen, A. K.-Y. Y.; Luscombe, C. K. Macromolecules 2009, 42 (20), 76707677.

(28) Senkovskyy, V.; Khanduyeva, N.; Komber, H.; Oertel, U.; Stamm, M.; Kuckling, D.; Kiriy, A. J. Am. Chem. Soc. 2007, 129 (20), 6626-6632.

(29) Khanduyeva, N.; Senkovskyy, V.; Beryozkina, T.; Bocharova, V.; Simon, F.; Nitschke, M.; Stamm, M.; Grötzschel, R.; Kiriy, A. Macromolecules 2008, 41 (20), 7383-7389.

(30) Bronstein, H. a.; Luscombe, C. K. J. Am. Chem. Soc. 2009, 131 (36), 12894-12895.

(31) Smeets, A.; Van den Bergh, K.; De Winter, J.; Gerbaux, P.; Verbiest, T.; Koeckelberghs, G. Macromolecules 2009, 42 (20), 7638-7641.

(32) Kempf, C. N.; Smith, K. a.; Pesek, S. L.; Li, X.; Verduzco, R. Polym. Chem. 2013, 4 (6), $2158-$ 2163.

(33) Doubina, N.; Paniagua, S. A.; Soldatova, A. V.; Jen, A. K. Y.; Marder, S. R.; Luscombe, C. K. Macromolecules 2011, 44 (3), 512-520.

(34) Kaul, E.; Senkovskyy, V.; Tkachov, R.; Bocharova, V.; Komber, H.; Stamm, M.; Kiriy, A. Macromolecules 2010, 43 (1), 77-81.

(35) Senkovskyy, V.; Tkachov, R.; Beryozkina, T.; Komber, H.; Oertel, U.; Horecha, M.; Bocharova, V.; Stamm, M.; Gevorgyan, S. a.; Krebs, F. C.; Kiriy, A. J. Am. Chem. Soc. 2009, 131 (45), 16445-16453.

(36) Doubina, N.; Stoddard, M.; Bronstein, H. a.; Jen, A. K.-Y.; Luscombe, C. K. Macromol. Chem. Phys. 2009, 210 (22), 1966-1972.

(37) Senkovskyy, V.; Sommer, M.; Tkachov, R.; Komber, H.; Huck, W. T. S.; Kiriy, A. Macromolecules 2010, 43 (23), 10157-10161.

(38) Hollinger, J.; Seferos, D. S. Macromolecules 2014, 47 (15), 5002-5009.

(39) Gao, L.-M.; Hu, Y.-Y.; Yu, Z.-P.; Liu, N.; Yin, J.; Zhu, Y.-Y.; Ding, Y.; Wu, Z.-Q. Macromolecules 2014, 47 (15), 5010-5018.

(40) Hidai, M.; Kashiwagi, T.; Ikeuchi, T.; Uchida, Y. J. Organomet. Chem. 1971, 30 (2), 279-282.

(41) Chavez, C. a.; Choi, J.; Nesterov, E. E. Macromolecules 2014, 47 (2), 506-516.

(42) Willot, P.; Moerman, D.; Leclère, P.; Lazzaroni, R.; Baeten, Y.; Van der Auweraer, M.; Koeckelberghs, G. Macromolecules 2014, 47 (19), 6671-6678.

(43) Verswyvel, M.; Monnaie, F.; Koeckelberghs, G. Macromolecules 2011, 44 (24), 9489-9498.

(44) Van den Bergh, K.; Willot, P.; Cornelis, D.; Verbiest, T.; Koeckelberghs, G. Macromolecules 2011, 44 (4), 728-735.

(45) Monnaie, F.; Brullot, W.; Verbiest, T.; De Winter, J.; Gerbaux, P.; Smeets, A.; Koeckelberghs, G. Macromolecules 2013, 46 (21), 8500-8508.

(46) Smeets, A.; Willot, P.; De Winter, J.; Gerbaux, P.; Verbiest, T.; Koeckelberghs, G. Macromolecules 2011, 44 (15), 6017-6025.

(47) Hardeman, T.; Willot, P.; Winter, J. De; Josse, T.; Gerbaux, P.; Shestakova, P.; Nies, E.; Koeckelberghs, G. J. Polym. Sci. Part A Polym. Chem. 2014, 52 (6), 804-809.

(48) Monnaie, F.; Verheyen, L.; De Winter, J.; Gerbaux, P.; Brullot, W.; Verbiest, T.; Koeckelberghs, G. Macromolecules 2015, 48 (24), 8752-8759.

(49) Monnaie, F.; Ceunen, W.; De Winter, J.; Gerbaux, P.; Cocchi, V.; Salatelli, E.; Koeckelberghs, G. Macromolecules 2015, 48 (1), 90-98.

(50) Van den Bergh, K.; De Winter, J.; Gerbaux, P.; Verbiest, T.; Koeckelberghs, G. Macromol. Chem. Phys. 2010, 212 (4), 328-335.

(51) Tkachov, R.; Senkovskyy, V.; Komber, H.; Sommer, J.-U.; Kiriy, A. J. Am. Chem. Soc. 2010, 132 (22), 7803-7810.

(52) Traina, C. A.; Bakus, R. C.; Bazan, G. C. J. Am. Chem. Soc. 2011, 133 (32), 12600-12607.

(53) Fronk, S. L.; Mai, C.-K.; Ford, M.; Noland, R. P.; Bazan, G. C. Macromolecules 2015, 48 (17), 6224-6232. 
(54) Pammer, F.; Jäger, J.; Rudolf, B.; Sun, Y. Macromolecules 2014, 47 (17), 5904-5912.

(55) Lee, S. R.; Bloom, J. W. G.; Wheeler, S. E.; McNeil, A. J. Dalton Trans. 2013, 42 (12), 4218 4222.

(56) Senkovskyy, V.; Tkachov, R.; Komber, H.; Sommer, M.; Heuken, M.; Voit, B.; Huck, W. T. S.; Kataev, V.; Petr, A.; Kiriy, A. J. Am. Chem. Soc. 2011, 133 (49), 19966-19970.

(57) Yokozawa, T.; Kohno, H.; Ohta, Y.; Yokoyama, A. Macromolecules 2010, 43 (17), 70957100.

(58) Yokozawa, T.; Suzuki, R.; Nojima, M.; Ohta, Y.; Yokoyama, A. Macromol. Rapid Commun. 2011, 32 (11), 801-806.

(59) Gutacker, A.; Lin, C.-Y.; Ying, L.; Nguyen, T.-Q.; Scherf, U.; Bazan, G. C. Macromolecules 2012, 45 (11), 4441-4446.

(60) Fischer, C. S.; Baier, M. C.; Mecking, S. J. Am. Chem. Soc. 2013, 135 (3), 1148-1154.

(61) Zhang, H.-H.; Xing, C.-H.; Hu, Q.-S.; Hong, K. Macromolecules 2015, 48 (4), 967-978.

(62) Zhang, H.; Xing, C.; Hu, Q. J. Am. Chem. Soc. 2012, 134 (32), 13156-13159.

(63) Elmalem, E.; Biedermann, F.; Johnson, K.; Friend, R. H.; Huck, W. T. S. J. Am. Chem. Soc. 2012, 134 (42), 17769-17777.

(64) Lee, J. K.; Ko, S.; Bao, Z. Macromol. Rapid Commun. 2012, 33 (10), 938-942.

(65) Kosaka, K.; Ohta, Y.; Yokozawa, T. Macromol. Rapid Commun. 2015, 36 (4), 373-377.

(66) Zhang, H.-H.; Hu, Q.-S.; Hong, K. Chem. Commun. 2015, 51 (80), 14869-14872.

(67) Verswyvel, M.; Hoebers, C.; De Winter, J.; Gerbaux, P.; Koeckelberghs, G. J. Polym. Sci. Part A Polym. Chem. 2013, 51 (23), 5067-5074.

(68) Elmalem, E.; Kiriy, A.; Huck, W. T. S. Macromolecules 2011, 44 (22), 9057-9061.

(69) Nanashima, Y.; Shibata, R.; Miyakoshi, R.; Yokoyama, A.; Yokozawa, T. J. Polym. Sci. Part A Polym. Chem. 2012, 50 (17), 3628-3640.

(70) Yuan, M.; Okamoto, K.; Bronstein, H. a.; Luscombe, C. K. ACS Macro Lett. 2012, 1 (3), 392395.

(71) Fischer, C. S.; Jenewein, C.; Mecking, S. Macromolecules 2015, 48 (3), 483-491.

(72) Senkovskyy, V.; Beryozkina, T.; Bocharova, V.; Tkachov, R.; Komber, H.; Lederer, A.; Stamm, M.; Severin, N.; Rabe, J. P.; Kiriy, A. Macromol. Symp. 2010, 291-292 (1), 17-25.

(73) Yang, X.; Loos, J. Macromolecules 2007, 40 (5), 1353-1362.

(74) Darling, S. B. J. Phys. Chem. B 2008, 112 (30), 8891-8895.

(75) Xin, H.; Reid, O. G.; Ren, G.; Kim, F. S.; Ginger, D. S.; Jenekhe, S. a. ACS Nano 2010, 4 (4), 1861-1872.

(76) Botiz, I.; Stingelin, N. Materials (Basel). 2014, 7 (3), 2273-2300.

(77) Sontag, S. K.; Marshall, N.; Locklin, J. Chem. Commun. (Camb). 2009, 23 (23), 3354-3356.

(78) Kang, S.; Ono, R. J.; Bielawski, C. W. J. Am. Chem. Soc. 2013, 135 (13), 4984-4987.

(79) Sontag, S. K.; Sheppard, G. R.; Usselman, N. M.; Marshall, N.; Locklin, J. Langmuir 2011, 27 (19), 12033-12041.

(80) Huddleston, N. E.; Sontag, S. K.; Bilbrey, J. A.; Sheppard, G. R.; Locklin, J. Macromol. Rapid Commun. 2012, 33 (24), 2115-2120.

(81) Khanduyeva, N.; Senkovskyy, V.; Beryozkina, T.; Horecha, M.; Stamm, M.; Uhrieh, C.; Riede, M.; Leo, K.; Kiriy, A. J. Am. Chem. Soc. 2009, 131 (1), 153-161.

(82) Beryozkina, T.; Boyko, K.; Khanduyeva, N.; Senkovskyy, V.; Horecha, M.; Oertel, U.; Simon, F.; Stamm, M.; Kiriy, A. Angew. Chemie Int. Ed. 2009, 48 (15), 2695-2698.

(83) Marshall, N.; Sontag, S. K.; Locklin, J. Macromolecules 2010, 43 (5), 2137-2144.

(84) Doubina, N.; Jenkins, J. L.; Paniagua, S. A.; Mazzio, K. A.; MacDonald, G. A.; Jen, A. K.-Y.; Armstrong, N. R.; Marder, S. R.; Luscombe, C. K. Langmuir 2012, 28 (3), 1900-1908.

(85) Senkovskyy, V.; Senkovska, I.; Kiriy, A. ACS Macro Lett. 2012, 1 (4), 494-498.

(86) Tkachov, R.; Senkovskyy, V.; Horecha, M.; Oertel, U.; Stamm, M.; Kiriy, A. Chem. Commun. (Camb). 2010, 46 (9), 1425-1427.

(87) Tkachov, R.; Senkovskyy, V.; Oertel, U.; Synytska, A.; Horecha, M.; Kiriy, A. Macromol. Rapid Commun. 2010, 31 (24), 2146-2150.

(88) Islam, M. A.; Purkait, T. K.; Mobarok, M. H.; Hoehlein, I. M. D.; Sinelnikov, R.; Iqbal, M.; Azulay, D.; Balberg, I.; Millo, O.; Rieger, B.; Veinot, J. G. C. Angew. Chemie Int. Ed. 2016, 55 (26), 7393-7397. 
(89) Boon, F.; Moerman, D.; Laurencin, D.; Richeter, S.; Guari, Y.; Mehdi, A.; Dubois, P.; Lazzaroni, R.; Clément, S. Langmuir 2014, 30 (38), 11340-11347.

(90) Roy, A.; Gao, J.; Bilbrey, J. A.; Huddleston, N. E.; Locklin, J. Langmuir 2014, 30 (34), 10465 10470.

(91) Youm, S. G.; Hwang, E.; Chavez, C. A.; Li, X.; Chatterjee, S.; Lusker, K. L.; Lu, L.; Strzalka, J.; Ankner, J. F.; Losovyj, Y.; Garno, J. C.; Nesterov, E. E. Chem. Mater. 2016, 28 (13), 47874804.

(92) Liu, C. L.; Lin, C. H.; Kuo, C. C.; Lin, S. T.; Chen, W. C. Prog. Polym. Sci. 2011, 36 (5), $603-$ 637.

(93) Tamao, K.; Sumitani, K.; Kumada, M. J. Am. Chem. Soc. 1972, 94 (12), 4374-4376.

(94) Mao, Y.; Wang, Y.; Lucht, B. L. J. Polym. Sci. Part A Polym. Chem. 2004, 42 (21), 55385547.

(95) Bryan, Z. J.; Smith, M. L.; McNeil, A. J. Macromol. Rapid Commun. 2012, 33 (9), 842-847.

(96) Loewe, R. S.; Khersonsky, S. M.; McCullough, R. D. Adv. Mater. 1999, 11 (3), 250-253.

(97) Lamps, J. P.; Catala, J. M. Macromolecules 2011, 44 (20), 7962-7968.

(98) Wu, S.; Huang, L.; Tian, H.; Geng, Y.; Wang, F. Macromolecules 2011, 44 (19), 7558-7567.

(99) Robert S. Loewe; Paul C. Ewbank; Jinsong Liu; Lei Zhai, A.; McCullough, R. D.; Loewe, R. S.; Ewbank, P. C.; Liu, J.; Zhai, L.; McCullough, R. D. Macromolecules 2001, 34 (13), 4324 4333.

(100) McCullough, R. D.; Lowe, R. D. J. Chem. Soc. Chem. Commun. 1992, 1 (1), 70-72.

(101) McCullough, R. D.; Williams, S. P.; Tristam-Nagle, S.; Jayaraman, M.; Ewbank, P. C.; Miller, L. Synth. Met. 1995, 69 (1-3), 279-282.

(102) Tamba, S.; Shono, K.; Sugie, A.; Mori, A. J. Am. Chem. Soc. 2011, 133 (25), 9700-9703.

(103) Tamba, S.; Tanaka, S.; Okubo, Y.; Meguro, H.; Okamoto, S.; Mori, A. Chem. Lett. 2011, 40 (4), 398-399.

(104) Heeney, M.; Zhang, W.; Crouch, D. J.; Chabinyc, M. L.; Gordeyev, S.; Hamilton, R.; Higgins, S. J.; McCulloch, I.; Skabara, P. J.; Sparrowe, D.; Tierney, S. Chem. Commun. (Camb). 2007, 5061-5063.

(105) Hollinger, J.; Jahnke, A. a.; Coombs, N.; Seferos, D. S. J. Am. Chem. Soc. 2010, 132 (25), 8546-8547.

(106) Ye, S.; Steube, M.; Carrera, E. I.; Seferos, D. S. Macromolecules 2016, 49 (5), 1704-1711.

(107) Wu, Z.-Q.; Radcliffe, J. D.; Ono, R. J.; Chen, Z.; Li, Z.; Bielawski, C. W. Polym. Chem. 2012, $3(4), 874-881$.

(108) Stefan, M. C.; Javier, A. E.; Osaka, I.; McCullough, R. D. Macromolecules 2009, 42 (1), 3032.

(109) Yokoyama, A.; Kato, A.; Miyakoshi, R.; Yokozawa, T. Macromolecules 2008, 41 (20), $7271-$ 7273.

(110) Lanni, E. L.; Locke, J. R.; Gleave, C. M.; McNeil, A. J. Macromolecules 2011, 44 (13), 51365145.

(111) Pammer, F.; Passlack, U. ACS Macro Lett. 2014, 3 (2), 170-174.

(112) Boon, F.; Hergué, N.; Deshayes, G.; Moerman, D.; Desbief, S.; De Winter, J.; Gerbaux, P.; Geerts, Y. H.; Lazzaroni, R.; Dubois, P. Polym. Chem. 2013, 4 (16), 4303-4307.

(113) Erdmann, T.; Back, J.; Tkachov, R.; Ruff, A.; Voit, B.; Ludwigs, S.; Kiriy, A. Polym. Chem. 2014, 5 (18), 5383-5390.

(114) Willot, P.; Govaerts, S.; Koeckelberghs, G. Macromolecules 2013, 46 (22), 8888-8895.

(115) Miyakoshi, R.; Shimono, K.; Yokoyama, A.; Yokozawa, T. J. Am. Chem. Soc. 2006, 128 (50), 16012-16013.

(116) Ohshimizu, K.; Takahashi, A.; Higashihara, T.; Ueda, M. J. Polym. Sci. Part A Polym. Chem. 2011, 49 (12), 2709-2714.

(117) Huang, L.; Wu, S.; Qu, Y.; Geng, Y.; Wang, F. Macromolecules 2008, 41 (22), 8944-8947.

(118) Javier, A. E.; Varshney, S. R.; McCullough, R. D. Macromolecules 2010, 43 (7), 3233-3237.

(119) Sui, A.; Shi, X.; Wu, S.; Tian, H.; Geng, Y.; Wang, F. Macromolecules 2012, 45 (13), 54365443.

(120) Nanashima, Y.; Yokoyama, A.; Yokozawa, T. J. Polym. Sci. Part A Polym. Chem. 2012, 50 (6), 1054-1061. 
(121) Bridges, C. R.; McCormick, T. M.; Gibson, G. L.; Hollinger, J.; Seferos, D. S. J. Am. Chem. Soc. 2013, 135 (35), 13212-13219.

(122) Bridges, C. R.; Yan, H.; Pollit, A. a; Seferos, D. S. ACS Macro Lett. 2014, 3 (7), 671-674.

(123) Wen, L.; Duck, B. C.; Dastoor, P. C.; Rasmussen, S. C. Macromolecules 2008, 41 (13), 45764578.

(124) Nojima, M.; Ohta, Y.; Yokozawa, T. J. Polym. Sci. Part A Polym. Chem. 2014, 52 (18), $2643-$ 2653.

(125) Bedi, A.; De Winter, J.; Gerbaux, P.; Koeckelberghs, G. J. Polym. Sci. Part A Polym. Chem. 2016, 54 (12), 1706-1712.

(126) Wu, S.; Sun, Y.; Huang, L.; Wang, J.; Zhou, Y.; Geng, Y.; Wang, F. Macromolecules 2010, 43 (10), 4438-4440.

(127) Lanni, E. L.; McNeil, A. J. J. Am. Chem. Soc. 2009, 131 (45), 16573-16579.

(128) Lanni, E. L.; McNeil, A. J. Macromolecules 2010, 43 (19), 8039-8044.

(129) Lee, S. R.; Bryan, Z. J.; Wagner, A. M.; McNeil, A. J. Chem. Sci. 2012, 3 (5), 1562-1566.

(130) Kohn, P.; Huettner, S.; Komber, H.; Senkovskyy, V.; Tkachov, R.; Kiriy, A.; Friend, R. H.; Steiner, U.; Huck, W. T. S.; Sommer, J.-U.; Sommer, M. J. Am. Chem. Soc. 2012, 134 (10), 4790-4805.

(131) Koeckelberghs, G.; Cornells, D.; Persoons, A.; Verbiest, T. Macromol. Rapid Commun. 2006, 27 (14), 1132-1136.

(132) Higashihara, T.; Goto, E. Polym. J. 2014, 46 (7), 381-390.

(133) Higashihara, T.; Goto, E.; Ueda, M. ACS Macro Lett. 2012, 1 (1), 167-170.

(134) Tkachov, R.; Senkovskyy, V.; Beryozkina, T.; Boyko, K.; Bakulev, V.; Lederer, A.; Sahre, K.; Voit, B.; Kiriy, A. Angew. Chemie - Int. Ed. 2014, 53 (9), 2402-2407.

(135) Brouwer, F.; Alma, J.; Valkenier, H.; Voortman, T. P.; Hillebrand, J.; Chiechi, R. C.; Hummelen, J. C. J. Mater. Chem. 2011, 21 (5), 1582.

(136) Jayakannan, M.; Lou, X.; Van Dongen, J. L. J.; Janssen, R. A. J. J. Polym. Sci. Part A Polym. Chem. 2005, 43 (7), 1454-1462.

(137) Jayakannan, M.; Van Dongen, J. L. J.; Janssen, R. A. J. Macromolecules 2001, 34 (16), 53865393.

(138) Kuivila, H. G.; Reuwer, J. F.; Mangravite, J. A. J. Am. Chem. Soc. 1964, 86 (13), 2666-2670.

(139) Carrillo, J. A.; Ingleson, M. J.; Turner, M. L. Macromolecules 2015, 48 (4), 979-986.

(140) Grisorio, R.; Mastrorilli, P.; Suranna, G. P. Polym. Chem. 2014, 5 (14), 4304-4310.

(141) Sui, A.; Shi, X.; Tian, H.; Geng, Y.; Wang, F. Polym. Chem. 2014, 5 (24), 7072-7080.

(142) Yokozawa, T.; Suzuki, R.; Nojima, M.; Ohta, Y.; Yokoyama, A. Macromol. Rapid Commun. 2011, 32 (11), 801-806.

(143) Nojima, M.; Ohta, Y.; Yokozawa, T. J. Am. Chem. Soc. 2015, 137 (17), 5682-5685.

(144) Chen, T. A.; Rieke, R. D. J. Am. Chem. Soc. 1992, 114 (25), 10087-10088.

(145) Bahri-Laleh, N.; Poater, A.; Cavallo, L.; Mirmohammadi, S. A. Dalton Trans. 2014, 43 (40), 15143-15150.

(146) Liu, W.; Tkachov, R.; Komber, H.; Senkovskyy, V.; Schubert, M.; Wei, Z.; Facchetti, A.; Neher, D.; Kiriy, A. Polym. Chem. 2014, 5 (10), 3404-3411.

(147) Suraru, S.-L.; Lee, J. A.; Luscombe, C. K. ACS Macro Lett. 2016, 5 (4), 533-536.

(148) Sanji, T.; Iyoda, T. J. Am. Chem. Soc. 2014, 136 (29), 10238-10241.

(149) Sanji, T.; Motoshige, A.; Komiyama, H.; Kakinuma, J.; Ushikubo, R.; Watanabe, S.; Iyoda, T. Chem. Sci. 2015, 6 (1), 492-496.

(150) Bonillo, B.; Swager, T. M. J. Am. Chem. Soc. 2012, 134 (46), 18916-18919.

(151) Nojima, M.; Saito, R.; Ohta, Y.; Yokozawa, T. J. Polym. Sci. Part A Polym. Chem. 2015, 53 (4), 543-551.

(152) Grisorio, R.; Suranna, G. P.; Mastrorilli, P. Chem. - A Eur. J. 2010, 16 (27), 8054-8061.

(153) Tu, G.; Li, H.; Forster, M.; Heiderhoff, R.; Balk, L. J.; Scherf, U. Macromolecules 2006, 39 (13), 4327-4331.

(154) Tu, G.; Li, H.; Forster, M.; Heiderhoff, R.; Balk, L. J.; Sigel, R.; Scherf, U. Small 2007, 3 (6), 1001-1006.

(155) Urien, M.; Erothu, H.; Cloutet, E.; Hiorns, R. C.; Vignau, L.; Cramail, H. Macromolecules 2008, 41 (19), 7033-7040. 
(156) Mulherin, R. C.; Jung, S.; Huettner, S.; Johnson, K.; Kohn, P.; Sommer, M.; Allard, S.; Scherf, U.; Greenham, N. C. Nano Lett. 2011, 11 (11), 4846-4851.

(157) Yu, X.; Xiao, K.; Chen, J.; Lavrik, N. V; Hong, K.; Sumpter, B. G.; Geohegan, D. B. ACS Nano 2011, 5 (5), 3559-3567.

(158) Asawapirom, U.; Güntner, R.; Forster, M.; Scherf, U. Thin Solid Films 2005, 477 (1-2), 48-52.

(159) Wu, P.; Ren, G.; Li, C.; Mezzenga, R.; Jenekhe, S. A. Macromolecules 2009, 42 (7), 23172320.

(160) Willot, P.; Teyssandier, J.; Dujardin, W.; Adisoejoso, J.; De Feyter, S.; Moerman, D.; Leclère, P.; Lazzaroni, R.; Koeckelberghs, G. RSC Adv. 2015, 5 (12), 8721-8726.

(161) Ge, J.; He, M.; Qiu, F.; Yang, Y. Macromolecules 2010, 43 (15), 6422-6428.

(162) Zhang, Y.; Tajima, K.; Hirota, K.; Hashimoto, K. J. Am. Chem. Soc. 2008, 130 (25), 7812 7813.

(163) Van den Bergh, K.; Huybrechts, J.; Verbiest, T.; Koeckelberghs, G. Chem. - A Eur. J. 2008, 14 (30), 9122-9125.

(164) Van den Bergh, K.; Cosemans, I.; Verbiest, T.; Koeckelberghs, G. Macromolecules 2010, 43 (8), 3794-3800.

(165) Chan, S.-H.; Lai, C.-S.; Chen, H.-L.; Ting, C.; Chen, C.-P. Macromolecules 2011, 44 (22), 8886-8891.

(166) Ouhib, F.; Khoukh, A.; Ledeuil, J. B.; Martinez, H.; Desbriéres, J.; Dagron-Lartigau, C. Macromolecules 2008, 41 (24), 9736-9743.

(167) Ouhib, F.; Hiorns, R. C.; de Bettignies, R.; Bailly, S.; Desbrières, J.; Dagron-Lartigau, C. Thin Solid Films 2008, 516 (20), 7199-7204.

(168) Clément, S.; Meyer, F.; De Winter, J.; Coulembier, O.; Vande Velde, C. M. L.; Zeller, M.; Gerbaux, P.; Balandier, J.-Y.; Sergeyev, S.; Lazzaroni, R.; Geerts, Y.; Dubois, P. J. Org. Chem. 2010, 75 (5), 1561-1568.

(169) Verswyvel, M.; Goossens, K.; Koeckelberghs, G. Polym. Chem. 2013, 4 (20), 5310.

(170) Wu, P.-T.; Ren, G.; Kim, F. S.; Li, C.; Mezzenga, R.; Jenekhe, S. a. J. Polym. Sci. Part A Polym. Chem. 2010, 48 (3), 614-626.

(171) Ho, C.; Liu, Y.; Lin, S.; Su, W. Macromolecules 2012, 45 (2), 813-820.

(172) Benanti, T. L.; Kalaydjian, A.; Venkataraman, D. Macromolecules 2008, 41 (22), 8312-8315.

(173) Ohshimizu, K.; Ueda, M. Macromolecules 2008, 41 (14), 5289-5294.

(174) Yokozawa, T.; Adachi, I.; Miyakoshi, R.; Yokoyama, A. High Perform. Polym. 2007, 19 (5-6), 684-699.

(175) Higashihara, T.; Ohshimizu, K.; Ryo, Y.; Sakurai, T.; Takahashi, A.; Nojima, S.; Ree, M.; Ueda, M. Polymer (Guildf). 2011, 52 (17), 3687-3695.

(176) Kim, J.; Siva, A.; Song, I. Y.; Park, T. Polymer (Guildf). 2011, 52 (17), 3704-3709.

(177) Kim, J.; Song, I. Y.; Park, T. Chem. Commun. 2011, 47 (16), 4697-4699.

(178) Song, I. Y.; Kim, J.; Im, M. J.; Moon, B. J.; Park, T. Macromolecules 2012, 45 (12), 50585068.

(179) Lin, Y.; Lim, J. A.; Wei, Q.; Mannsfeld, S. C. B.; Briseno, A. L.; Watkins, J. J. Chem. Mater. 2012, $24(3), 622-632$.

(180) Li, F.; Yang, J.; Qin, Y. J. Polym. Sci. Part A Polym. Chem. 2013, 51 (16), 3339-3350.

(181) Miyanishi, S.; Zhang, Y.; Hashimoto, K.; Tajima, K. Macromolecules 2012, 45 (16), 64246437.

(182) Li, F.; Yager, K. G.; Dawson, N. M.; Yang, J.; Malloy, K. J.; Qin, Y. Macromolecules 2013, 46 (22), 9021-9031.

(183) Monnaie, F.; Van Den Eede, M. P.; Koeckelberghs, G. Macromolecules 2015, 48 (22), $8121-$ 8127.

(184) Miyakoshi, R.; Yokoyama, A.; Yokozawa, T. Chem. Lett. 2008, 37 (10), 1022-1023.

(185) Wu, S.; Bu, L.; Huang, L.; Yu, X.; Han, Y.; Geng, Y.; Wang, F. Polymer (Guildf). 2009, 50 (26), 6245-6251.

(186) Yokozawa, T.; Kohno, H.; Ohta, Y.; Yokoyama, A. Macromolecules 2010, 43 (17), 7095 7100.

(187) Javier, A. E.; Varshney, S. R.; McCullough, R. D. Macromolecules 2010, 43 (7), 3233-3237.

(188) Angiolini, L.; Brazzi, A.; Salatelli, E.; Van den Bergh, K.; Koeckelberghs, G. Macromol. 
Chem. Phys. 2013, 214 (8), 934-942.

(189) Kozycz, L. M.; Gao, D.; Hollinger, J.; Seferos, D. S. Macromolecules 2012, 45 (14), $5823-$ 5832.

(190) Palermo, E. F.; McNeil, A. J. Macromolecules 2012, 45 (15), 5948-5955.

(191) Hollinger, J.; DiCarmine, P. M.; Karl, D.; Seferos, D. S. Macromolecules 2012, 45 (9), 37723778.

(192) Goto, E.; Nakamura, S.; Kawauchi, S.; Mori, H.; Ueda, M.; Higashihara, T. J. Polym. Sci. Part A Polym. Chem. 2014, 52 (16), 2287-2296.

(193) Verswyvel, M.; Steverlynck, J.; Hadj Mohamed, S.; Trabelsi, M.; Champagne, B.; Koeckelberghs, G. Macromolecules 2014, 47 (14), 4668-4675.

(194) Lee, S. K.; Lee, W. H.; Cho, J. M.; Park, S. J.; Park, J. U.; Shin, W. S.; Lee, J. C.; Kang, I. N.; Moon, S. J. Macromolecules 2011, 44 (15), 5994-6001.

(195) Li, Y. Acc. Chem. Res. 2012, 45 (5), 723-733.

(196) Zhang, Z.; Wang, J. J. Mater. Chem. 2012, 22 (10), 4178.

(197) Ono, R. J.; Kang, S.; Bielawski, C. W. Macromolecules 2012, 45 (5), 2321-2326.

(198) Todd, A. D.; Bielawski, C. W. ACS Macro Lett. 2015, 4 (11), 1254-1258.

(199) Bronstein, H.; Hurhangee, M.; Fregoso, E. C.; Beatrup, D.; Soon, Y. W.; Huang, Z.; Hadipour, A.; Tuladhar, P. S.; Rossbauer, S.; Sohn, E. H.; Shoaee, S.; Dimitrov, S. D.; Frost, J. M.; Ashraf, R. S.; Kirchartz, T.; Watkins, S. E.; Song, K.; Anthopoulos, T.; Nelson, J.; Rand, B. P.; Durrant, J. R.; McCulloch, I. Chem. Mater. 2013, 25 (21), 4239-4249.

(200) Qiu, Y.; Fortney, A.; Tsai, C. H.; Baker, M. A.; Gil, R. R.; Kowalewski, T.; Noonan, K. J. T. ACS Macro Lett. 2016, 5 (3), 332-336.

(201) Tsai, C.-H.; Fortney, A.; Qiu, Y.; Gil, R. R.; Yaron, D.; Kowalewski, T.; Noonan, K. J. T. J. Am. Chem. Soc. 2016, 138 (21), 6798-6804.

(202) Hardeman, T.; Koeckelberghs, G. Macromolecules 2015, 48 (19), 6987-6993.

(203) Willot, P.; Steverlynck, J.; Moerman, D.; Leclère, P.; Lazzaroni, R.; Koeckelberghs, G. Polym. Chem. 2013, 4 (9), 2662-2671.

(204) Locke, J. R.; McNeil, A. J. Macromolecules 2010, 43 (21), 8709-8710.

(205) Palermo, E. F.; van der Laan, H. L.; McNeil, A. J. Polym. Chem. 2013, 4 (17), 4606-4611.

(206) Wu, P.-T. T.; Ren, G.; Jenekhe, S. A. Macromolecules 2010, 43 (7), 3306-3313.

(207) Hollinger, J.; Sun, J.; Gao, D.; Karl, D.; Seferos, D. S. Macromol. Rapid Commun. 2013, 34 (5), 437-441.

(208) Pollit, A. a.; Bridges, C. R.; Seferos, D. S. Macromol. Rapid Commun. 2015, 36 (1), 65-70.

(209) Hardeman, T.; Koeckelberghs, G. Macromolecules 2014, 47 (24), 8618-8624.

(210) Miyakoshi, R.; Yokoyama, A.; Yokozawa, T. Macromol. Rapid Commun. 2004, 25 (19), $1663-1666$.

(211) Lohwasser, R. H.; Thelakkat, M. Macromolecules 2011, 44 (9), 3388-3397.

(212) Malika, J. E. L.; Sauvé, G.; McCullough, R. D. Adv. Mater. 2004, 16 (12), 1017-1019.

(213) Jeffries-El, M.; Sauvé, G.; McCullough, R. D. Macromolecules 2005, 38 (25), 10346-10352.

(214) Kochemba, W. M.; Pickel, D. L.; Sumpter, B. G.; Chen, J.; Kilbey, S. M. Chem. Mater. 2012, 24 (22), 4459-4467.

(215) Bürger, H.; Wannagat, U. Monatshefte für Chemie 1964, 95 (4), 1099-1102.

(216) Iovu, M. C.; Jeffries-EL, M.; Sheina, E. E.; Cooper, J. R.; McCullough, R. D. Polymer (Guildf). 2005, 46 (19), 8582-8586.

(217) Dai, C.; Yen, W.; Lee, Y.; Ho, C.; Su, W. J. Am. Chem. Soc. 2007, 129 (36), 11036-11038.

(218) Higashihara, T.; Ohshimizu, K.; Hirao, A.; Ueda, M. Macromolecules 2008, 41 (24), 95059507.

(219) Higashihara, T.; Takahashi, A.; Tajima, S.; Jin, S.; Rho, Y.; Ree, M.; Ueda, M. Polym. J. 2010, $42(1), 43-50$.

(220) Moon, H. C.; Anthonysamy, A.; Lee, Y.; Kim, J. K. Macromolecules 2010, 43 (4), 1747-1752.

(221) Takahashi, A.; Rho, Y.; Higashihara, T.; Ahn, B.; Ree, M.; Ueda, M. Macromolecules 2010, 43 (11), 4843-4852.

(222) Kochemba, W. M.; Kilbey, S. M.; Pickel, D. L. J. Polym. Sci. Part A Polym. Chem. 2012, 50 (14), 2762-2769.

(223) Okamoto, K.; Luscombe, C. K. Chem. Commun. 2014, 50 (40), 5310-5312. 
(224) Chen, C.-M.; Jen, T.-H.; Chen, S.-A. ACS Appl. Mater. Interfaces 2015, 7 (37), 20548-20555.

(225) Fujita, H.; Michinobu, T.; Tokita, M.; Ueda, M.; Higashihara, T. Macromolecules 2012, 45 (24), 9643-9656.

(226) van As, D.; Subbiah, J.; Jones, D. J.; Wong, W. W. H. Macromol. Chem. Phys. 2016, 217 (3), 403-413.

(227) Deckers, S.; Steverlynck, J.; Willot, P.; Vandendriessche, S.; Koeckelberghs, G.; Asselberghs, I.; Verbiest, T.; Van Der Veen, M. A. J. Phys. Chem. C 2015, 119 (32), 18513-18517.

(228) Shen, J.; Masaoka, H.; Tsuchiya, K.; Ogino, K. Polym. J. 2008, 40 (5), 421-427.

(229) Pu, K. Y.; Chen, Y.; Qi, X. Y.; Qin, C. Y.; Chen, Q. Q.; Wang, H. Y.; Deng, Y.; Fan, Q. L.; Huang, Y. Q.; Liu, S. J.; Wei, W.; Peng, B.; Huang, W. J. Polym. Sci. Part A Polym. Chem. 2007, 45 (16), 3776-3787.

(230) Wang, J.; Lu, C.; Mizobe, T.; Ueda, M.; Chen, W. C.; Higashihara, T. Macromolecules 2013, $46(5), 1783-1793$.

(231) Zeigler, D. F.; Mazzio, K. A.; Luscombe, C. K. Macromolecules 2014, 47 (15), 5019-5028.

(232) Steverlynck, J.; De Cattelle, A.; De Winter, J.; Gerbaux, P.; Koeckelberghs, G. J. Polym. Sci. Part A Polym. Chem. 2016, 54 (9), 1252-1258.

(233) Steverlynck, J.; De Winter, J.; Gerbaux, P.; Lazzaroni, R.; Leclère, P.; Koeckelberghs, G. Macromolecules 2015, 48 (24), 8789-8796.

(234) Hadjichristidis, N.; Pitsikalis, M.; Pispas, S.; Iatrou, H. Chem. Rev. 2001, 101 (12), 3747-3792.

(235) Ying, L.; Zalar, P.; Collins, S. D.; Chen, Z.; Mikhailovsky, A. A.; Nguyen, T. Q.; Bazan, G. C. Adv. Mater. 2012, 24 (48), 6496-6501.

(236) Zhang, H. H.; Xing, C. H.; Hu, Q. S. J. Am. Chem. Soc. 2012, 134 (32), 13156-13159.

(237) Kromer, J.; Rios-Carreras, I.; Fuhrmann, G.; Musch, C.; Wunderlin, M.; Debaerdemaeker, T.; Mena-Osteritz, E.; Bauerle, P. Angew. Chemie - Int. Ed. 2000, 39 (19), 3481-3486.

(238) Mayor, M.; Didschies, C. Angew. Chemie - Int. Ed. 2003, 42 (27), 3176-3179.

(239) Zhang, F.; Götz, G.; Winkler, H. D. F.; Schalley, C. A.; Bäuerle, P. Angew. Chemie - Int. Ed. 2009, 48 (36), 6632-6635.

(240) Hensel, V.; Lützow, K.; Jacob, J.; Gessler, K.; Saenger, W.; Schlüter, A.-D. Angew. Chem. Int. Ed. Engl. 1997, 36 (23), 2654-2656.

(241) Hensel, V.; Schlüter, A. D. Chem. - A Eur. J. 1999, 5 (2), 421-429.

(242) Jasti, R.; Bhattacharjee, J.; Neaton, J. B.; Bertozzi, C. R. J. Am. Chem. Soc. 2008, 130 (52), 17646-17647.

(243) Kayahara, E.; Sakamoto, Y.; Suzuki, T.; Yamago, S. Org. Lett. 2012, 14 (13), 3284-3287.

(244) Iwamoto, T.; Watanabe, Y.; Sakamoto, Y.; Suzuki, T.; Yamago, S. J. Am. Chem. Soc. 2011, $133(21), 8354-8361$.

(245) Coulembier, O.; Deshayes, G.; Surin, M.; De Winter, J.; Boon, F.; Delcourt, C.; Leclère, P.; Lazzaroni, R.; Gerbaux, P.; Dubois, P. Polym. Chem. 2013, 4 (2), 237-241.

(246) Liu, J.; McCullough, R. D. Macromolecules 2002, 35 (27), 9882-9889.

(247) Surin, M.; Coulembier, O.; Tran, K.; Winter, J. De; Leclère, P.; Gerbaux, P.; Lazzaroni, R.; Dubois, P. Org. Electron. 2010, 11 (5), 767-774.

(248) Steverlynck, J.; Leysen, P.; Koeckelberghs, G. J. Polym. Sci. Part A Polym. Chem. 2015, 53 (1), 79-84.

(249) Karpe, S.; Cravino, A.; Frère, P.; Allain, M.; Mabon, G.; Roncali, J. Adv. Funct. Mater. 2007, 17 (7), 1163-1171.

(250) Ma, C. Q.; Mena-Osteritz, E.; Debaerdemaeker, T.; Wienk, M. M.; Janssen, R. A. J.; Bäuerle, P. Angew. Chemie - Int. Ed. 2007, 46 (10), 1679-1683.

(251) Richter, T. V.; Link, S.; Hanselmann, R.; Ludwigs, S. Macromol. Rapid Commun. 2009, 30 (15), 1323-1327.

(252) Xu, M. H.; Pu, L. Tetrahedron Lett. 2002, 43 (36), 6347-6350.

(253) Scheuble, M.; Richter, T. V.; Goll, M.; Link, S.; López Navarrete, J. T.; Ruff, A.; Ruiz Delgado, M. C.; Ludwigs, S. Polym. Chem. 2014, 5 (23), 6824-6833.

(254) Okamoto, K.; Housekeeper, J. B.; Michael, F. E.; Luscombe, C. K. Polym. Chem. 2013, 4 (12), 3499-3506.

(255) Tu, G.; Bilge, A.; Adamczyk, S.; Forster, M.; Heiderhoff, R.; Balk, L. J.; Mühlbacher, D.; Morana, M.; Koppe, M.; Scharber, M. C.; Choulis, S. A.; Brabec, C. J.; Scherf, U. Macromol. 
Rapid Commun. 2007, 28 (17), 1781-1785.

(256) Huang, W.; Su, L.; Bo, Z. J. Am. Chem. Soc. 2009, 131 (30), 10348-10349.

(257) Bo, Z.; Schlüter, A. D. Chem. Commun. 2003, 2354-2355.

(258) Xue, Z.; Finke, A. D.; Moore, J. S. Macromolecules 2010, 43 (22), 9277-9282.

(259) Segawa, Y.; Higashihara, T.; Ueda, M. Polym. Chem. 2013, 4 (4), 1208-1215.

(260) Kim, H. J.; Lee, Y. J.; Hwang, S. S.; Choi, D. H.; Yang, H.; Baek, K. Y. J. Polym. Sci. Part A Polym. Chem. 2011, 49 (19), 4221-4226.

(261) Wang, F.; Rauh, R. D.; Rose, T. L. J. Am. Chem. Soc. 1997, 119 (45), 11106-11107.

(262) Wang, F.; Wilson, M. S.; Rauh, R. D.; Schottland, P.; Thompson, B. C.; Reynolds, J. R. Macromolecules 2000, 33 (6), 2083-2091. 